International Journal of Computer Vision 17(3), 241-272 (1996)

(C) 1996 Kluwer Academic Publishers. Manufactured in The Netherlands.

\title{
Detection of Diffuse and Specular Interface Reflections and Inter-Reflections by Color Image Segmentation
}

\author{
RUZENA BAJCSY \\ GRASP Laboratory, Department of Computer and Information Science, University of Pennsylvania, \\ Philadelphia, PA 19104 \\ SANG WOOK LEE \\ Dept. of Electrical Engineering and Computer Science, Univ. of Michigan, 1101 Beal Ave., Ann Arbor, MI 48109 \\ ALEŠ LEONARDIS \\ Faculty of Electrical Engineering and Computer Science, Trzaska c. 25, 61001 Ljubljana, Slovenia
}

Received June 23, 1992; Revised September 10, 1992; Accepted November 23, 1993

\begin{abstract}
We present a computational model and algorithm for detecting diffuse and specular interface reflections and some inter-reflections. Our color reflection model is based on the dichromatic model for dielectric materials and on a color space, called $\mathbf{S}$ space, formed with three orthogonal basis functions. We transform color pixels measured in RGB into the $\mathbf{S}$ space and analyze color variations on objects in terms of brightness, hue and saturation which are defined in the $\mathbf{S}$ space. When transforming the original RGB data into the $\mathbf{S}$ space, we discount the scene illumination color that is estimated using a white reference plate as an active probe. As a result, the color image appears as if the scene illumination is white. Under the whitened illumination, the interface reflection clusters in the $\mathbf{S}$ space arc all aligned with the brightness direction. The brightncss, huc and saturation values exhibit a more direct correspondence to body colors and to diffuse and specular interface reflections, shading, shadows and interreflections than the RGB coordinates. We exploit these relationships to segment the color image, and to separate specular and diffuse interface reflections and some inter-reflections from body reflections. The proposed algorithm is efficacious for uniformly colored dielectric surfaces under singly colored scene illumination. Experimental results conform to our model and algorithm within the limitations discussed.
\end{abstract}

\section{Keywords:}

\section{Introduction}

Detection of specularities from Lambertian reflections is an important real-world problem in computational vision. Most vision algorithms are based on the assumption that visually observable surfaces consist only of Lambertian reflection. Specularity is one of the major hindrances to vision tasks such as image segmentation, object recognition and shape or structure determination. Once successfully detected and separated from Lambertian reflections, however, the specularity is no longer a problem, but extra information potentially as useful as Lambertian reflection. Specularity can provide information about the surface roughness of an object, and the local shape of objects separately from Lambertian reflection (Blake, 1985; Healey and Binford, 1988b). Therefore, it is desirable to have algorithms for estimating reflectance properties as a very carly stage or an integral part of many visual processes.

Recently the use of physical information for disambiguating different reflection types has been brought to the attention of the computer vision field (Kanade and Ikeuchi, 1991). There have been five principal approaches so far in solving the problem of specularity detection or separation: using (1) a single grey-scale 
image, (2) different light directions, (3) polarization, (4) color, (5) color and polarization, and (6) color images from multiple viewpoints.

There has been an approach to the detection of specularity with a single gray-level image using the Lambertian constraints by Brelstaff and Blake (1988). Although successful detection of some apparcnt specularities was demonstrated, the problem is physically underconstrained, given only a single gray-level image. The recent photometric-stereo-type approaches consider the specular and Lambertian reflectance properties for obtaining object shape using more than two light directions. Those approaches were introduced by Coleman and Jain (1982), Nayar et al. (1990a), Park and Tou (1990) and Tagare and deFigueiredo (1990; 1991). The basic technique of the pholometricstereo-type approaches is the switching of illumination sources. Therefore, application is restricted to darkroom environments where the direction and collimation of illumination can be strictly controlled.

Wolff proposed a method of detecting specularities from dielectrics and metals using the analysis of the polarization of reflected light (Wolff, 1991). Wolff also suggested a method of classifying material types, metal or dielectric, using the degree of polarization in the detected specularities. Physical properties of color have also been exploited for identifying specular reflections. The dichromatic model (Shafer, 1985) suggested by Shafer has been the key model to many highlight detection algorithms using color, such as the ones by Shafer et al., (1990), Gershon (1987), Healey and Binford (1988), and Bajcsy et al. (1990). Recently Nayar et al. (1993) suggested a method for separating reflection components using the dichromatic model and polarization. The use of color and multiple views has been proposed by Lee (1991) and Lee and Bajcsy (1992). The algorithm, called spectral differencing, detects specularities from both dielectrics and metals by finding color differences from different viewpoints. The algorithm does not rely on the dichromatic model.

The intended contribution of our model and algorithm presented in this paper is to detect and separate diffuse interface reflections and some inter-reflections. There are two physically different types of reflections for inhomogeneous dielectric materials according to the dichromatic model proposed by Shafer: interface reflection and body reflection (Nassau, 1983; Shafer, 1985). Body reflection is very diffuse and often modeled as perfectly diffuse reflection, i.e., Lambertian reflection (Horn, 1986). Interface reflection, on the other hand, can be specular or diffuse depending on surface roughness and illumination. Diffuse interface reflections result from a rough surface of an object. When illumination is extended and diffuse, interface reflections can also appear diffusely even from a smooth surface. While interface reflection from a smooth surface and collimated light exhibits a sharp specular highlight, diffuse interface reflection looks softer, and very diffuse interface reflection produces lustrous sheen.

Diffuse interface reflection as well as specular interface reflection can be a source of confusion for many vision algorithms. However, the previous algorithms, whether based on photometric stereo, polarization, color, or color with multiple views, tend to detect or separate specular interface reflections, and the problem of detecting or separating diffuse interface reflections has not been well addressed. In this paper, we propose a new algorithm that can detect and separate diffuse and well as specular interface reflections from body reflections from dielectric materials. We also present a method for detecting some inter-reflections. Inter-reflection is the multiple reflection between object surfaces, and it is often observed when there are many objects close to each other. In many cases, interreflections are diffuse, since light sources for interreflections are adjacent objects which are often extended and diffuse sources of light.

In this paper, we present a color reflection model in a three-dimensional color space, called $\mathbf{S}$ space, based on three orthogonal basis functions. The dichromatic model by Shafer is used as an essential guide in physical modeling, and rough surface and extended illumination are considered in our model in order to analyze the color variation due to diffuse interface reflection and inter-reflection. We analyze color variations on objects in terms of brightness, hue and saturation which are defined in the $\mathbf{S}$ space, and we discount the scene illumination color that is estimated using a white reference plate. The $\mathbf{S}$ space and the compensation for illumination color facilitate the separation of diffuse interface reflections and inter-reflections from body reflections. We analyze inter-reflections in four components based on our previous work: interface-interface, interface-body, body-interface and body-body reflectiuns (Bajcsy et al., 1990). We also propose an algorithm for detecting some of the inter-reflections.

We describe reflection models and a separation algorithm in three sections of this paper, one discussing basic reflection models our work is based on and the other two presenting our model and algorithm. In Section 2, 
basic color models for light reflections are summarized. The spectral scene radiance model is presented in Section 3 for analyzing the variation of interface and body reflection colors in the $\mathbf{S}$ space. We also discuss color variation of illumination due to multiple objects to derive color models for inter-reflections and shadows. Section 4 presents our scparation algorithm based on the spectral scene radiance model, and discusses the assumptions imposed on object surfaces and illumination. Experimental results in Section 5 show the efficacy of our algorithm for detecting and separating specular and diffuse interface reflections and inter-reflections.

\section{Spectral Reflection Model}

Basic physical models for the interpretation of lightsurface interaction and sensing are crucial for developing the model and the algorithm proposed in this paper. Our work is based on some basic reflection models that are generally well accepted in computer vision for their good approximation of physical phenomena (Healey, 1989; Lee et al., 1990; Nayar et al., 1991; Shafer, 1985; Tagare and deFigueiredo, 1991; Torrance and Sparrow, 1967).

Materials are commonly classified as metals and dielectrics depending on their electrical (or optical) properties. Materials are also divided into two classes as optically homogeneous and inhomogeneous materials (Healey, 1989; Shafer, 1985). Optically homogeneous materials, such as metals and glass, have a homogeneous index of refraction throughout the material. Optically inhomogeneous materials, such as opaque plastics, paper and paints, are composed of more than one material with different refractive indices, usually of a vchicle diclectric matcrial and embedded pigment particles. In this paper, models are established only for the reflected light from opaque inhomogeneous dielectric materials, and homogeneous materials such as metals and transparent dielectrics are not considered. For a color model of metals, see (Healey, 1989).

\subsection{Reflection Types}

When scene illumination is singly colored, the reflected light from a surface patch is the product of illumination and surface reflection as (Lee, 1991)

$$
L_{r}(\lambda)=e(\lambda) s(\lambda)
$$

where $s(\lambda)$ is a spectral power distribution (SPD) of surface reflection, $e(\lambda)$ is a SPD of illumination and $\lambda$ is the wavelength of light. We use Shafer's nomenclature for two physically different types of surface reflections for inhomogeneous dielectric materials: interface reflection and body reflection (Nassau, 1983; Shafer, 1985). The interface reflection occurs at the interface of two different materials, such as air and material surface. The ratio of the reflected to the refracted light is determined by the angle of incidence and the refractive indices of the media. The refracted light going into a subsurface is scattered and absorbed by the internal pigments, and some of the scattered light is re-emitted randomly resulting in the body reflection. Depending on the pigment material and distribution, the reflected light undergoes a spectral change. For a detailed discussion of interface and body reflections, see (Shafer, 1985). Note that we do not use "surface reflection" for denoting the interface reflection since, in a wider sense, it means all the reflections from surface and sub-surface. In this paper, "surface reflection" is used in the wider sense. The reflection types are summarized in Fig. 1.

The surface reflection $s(\lambda)$ can be expressed as the linear combination of interface and body reflections with the different geometric weighting factors, given as (Healey, 1989; Shafer, 1985)

$$
s(\lambda)=\rho_{S}(\lambda) G_{S}\left(\theta_{r}, \phi_{r}\right)+\rho_{B}(\lambda) G_{B},
$$

where $\rho_{S}(\lambda)$ and $\rho_{B}(\lambda)$ are the interface and the body reflectances, i.e., Fresnel reflectance and albedo, respectively, $\left(\theta_{r}, \phi_{r}\right)$ is the viewing direction, and $G_{S}\left(\theta_{r}, \phi_{r}\right)$ and $G_{B}$ are the geometric weighting factors for interface and body reflections, respectively. Note that the variables $\left(\theta_{r}, \phi_{r}\right)$ are omitted in $s(\lambda)$. The observation of the interface reflection is highly dependent both on the viewer and on the illumination directions. When the body reflection is modeled as Lambertian reflection, $G_{B}$ is determined by illumination directions, but independent of the viewing angle $\left(\theta_{r}, \phi_{r}\right)$. For nonLambertian models of the body reflection, see (Healey, 1989; Oren and Nayar, 1993; Wolff, 1993). Since the refractive indices of dielectric material are nearly independent of wavelength over the visible range of light ( $400 \mathrm{~nm}$ to $700 \mathrm{~nm}$ of wavelength), interface reflectance of dielectrics can be well approximated as flat spectrum as shown in Fig. 1, and this approximation has been tested and called the neutral interface reflection (NIR) model by Lee et al. (1990) and Healey (1989).

Reflections can also be categorized as specular and diffuse reflections depending on the degree of diffusion in the reflected light direction. Body reflection is very diffuse and as mentioned earlier, often modeled 


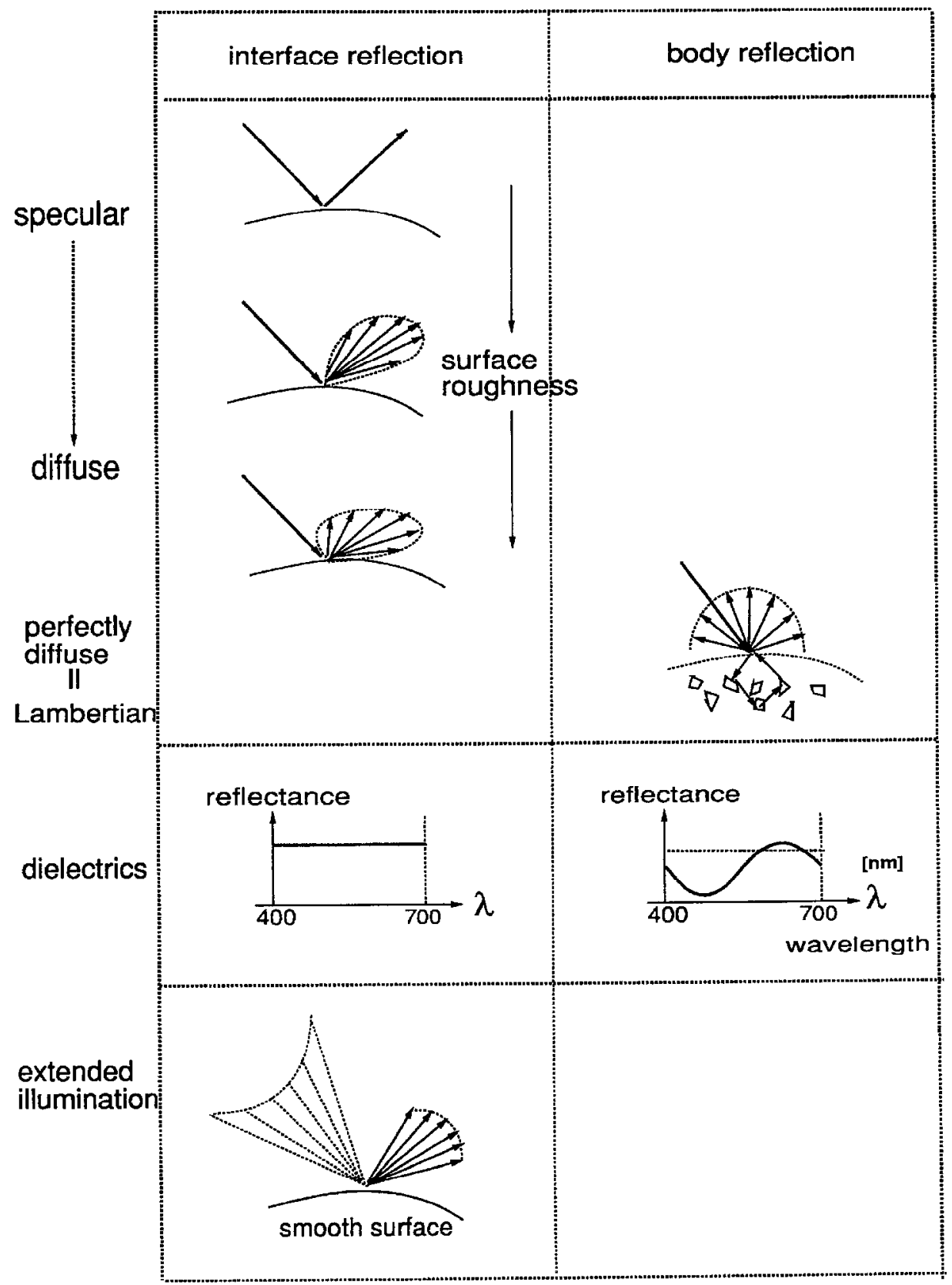

Figure 1. Reflection model. 
as a perfectly diffuse reflection, i.e., Lambertian reflection. The interface reflection, however, can be specular or diffuse depending on the surface roughness. A highly polished surface and collimated light can produce a specularity which we call a "specular interface reflection". As surfaces are roughened, the interface reflection becomes diffuse and looks softer, and we call this "diffuse interface reflection". Very diffuse interface reflections look lustrous. In general, the rougher a surface is, the more diffuse the reflected light is as illustrated in Fig. 1. There have been some models that describe the scattering of light by rough surfaces, such as those by Beckman and Spizzichino (1963) and Torrance and Sparrow (1967). The geometric modeling by Torrance and Sparrow is widely accepted in computer vision and graphics as a good approximation of the physical phenomenon. For detailed discussions of specular and diffuse reflections, see (Nayar et al., 1991; Tagare and deFigueiredo, 1991; Wolff, 1989).

Diffusion in the interface reflections can result from extended and diffuse illumination as shown in Fig. 1. When illumination is extended and diffuse, the incident angle of light to a surface patch is extended and interface reflections at even a smooth surface appear diffusely. When the surface is rough, the reflection is more diffuse. The diffuse interface reflection that results from extended and diffuse illumination is often indistinguishable in color and in spatial appearance from the diffuse interface reflection from a rough surface and collimated illumination.

It is only when the scene illumination is singly colored that the scene radiance $L_{r}$ is given as the product of the scene illumination $e(\lambda)$ and the surface reflection $s(\lambda)$ (Lee et al., 1990; Lee, 1991). For multiply colored illumination, we use the addition of scene radiance from differently colored illumination sources

$$
L_{r}(\lambda)=e_{1}(\lambda) s_{1}(\lambda)+e_{2}(\lambda) s_{2}(\lambda)+\cdots
$$

for analyzing reflection components.

\subsection{Representation of Color}

In analyzing the scene radiance $L_{r}(\lambda)$, we use a color space based on three orthogonal basis functions. We also discount the scene illumination color in an image since this simplifies our analysis. In this subsection, wc describe our color representation and a simple method for discounting illumination color using a reference patch. A detailed discussion of the color space and the analysis of various surface reflections in the space will be given in Section 3 .
Scene radiance from an object point can be represented as a weighted sum of basis functions expressed as:

$$
L_{r}(\lambda)=\sum_{i=0}^{2} \gamma_{i} S_{i}(\lambda)
$$

where $S_{i}(\lambda)$ are the basis functions and $\gamma_{i}$ 's are the scalar weighting factors. It is the distribution of the color vector $\gamma=\left[\gamma_{0}, \gamma_{1}, \gamma_{2}\right]^{T}$ in the color space $\left(S_{0}, S_{1}, S_{2}\right)$ that we use in this paper for analyzing scene radiance. The color vector $\gamma$ can be obtained from the color sensor responses. $\overline{\mathrm{We}}$ use $\mathrm{R}, \mathrm{G}$ and $\mathrm{B}$ filters and a CCD camera. The sensor responses are given by

$$
q_{k}=\int_{\lambda_{1}}^{\lambda_{2}} L_{r}(\lambda) Q_{k}(\lambda) d \lambda
$$

where $Q_{k}(\lambda)$ and $q_{k}$ for $k=0,1,2$ are the spectral response of the $k$ th filter, and the camera output through the $k$ th filter respectively. The wavelengths $\lambda_{1}=400 \mathrm{~nm}$ and $\lambda_{2}=700 \mathrm{~nm}$ cover the range of the visible spectrum. The relationship between the sensor response and $\underline{\gamma}$ is given as

$$
\underline{\mathbf{q}}=\underline{\mathbf{\Lambda}} \underline{\gamma}, \quad \text { or } \quad \underline{\gamma}=\underline{\mathbf{V}} \underline{\mathbf{q}},
$$

where $\mathbf{q}=\left[q_{0}, q_{1}, q_{2}\right]^{T}, \underline{\mathbf{V}}=\underline{\Lambda}^{-1}$, and the elements of $\underline{\Lambda}$ in the $k$ th row and $i$ th column are given by

$$
\Lambda_{k i}=\int_{\lambda_{1}}^{\lambda_{2}} S_{i}(\lambda) Q_{k}(\lambda) d \lambda .
$$

To obtain $\underline{\gamma}$, we need to know the spectral responses of color sensors. Figure 2(c) shows the spectral response of the three filters we use: $Q_{0}(\lambda), Q_{1}(\lambda)$ and $Q_{2}(\lambda)$. The RGB curves were measured with a spectrophotometer and they include the spectral responses of our CCD camera and infrared cut-off filter (shown in Fig. 2(a)), and coated camera lens (shown in Fig. 2(b)).

The color vector $\gamma$ contains the colors of both surface reflection and illumination. As mentioned earlier, since neutral illumination facilitates our color analysis, we discount scene illumination color using a reference patch. Below we briefly describe this process.

For representing surface reflection and illumination, we also use a set of three basis functions:

$$
s(\lambda)=\sum_{i=0}^{2} \sigma_{i} S_{i}(\lambda), \quad e(\lambda)=\sum_{j=0}^{2} \varepsilon_{j} E_{j}(\lambda),
$$




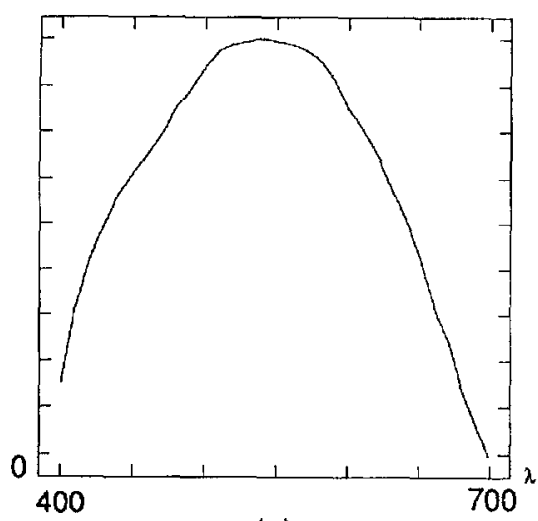

(a)

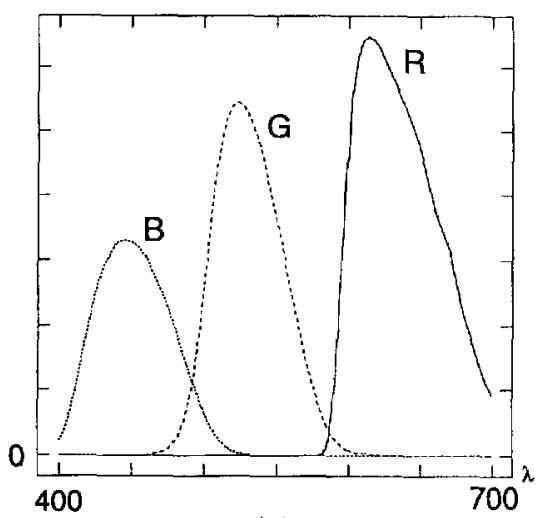

(c)

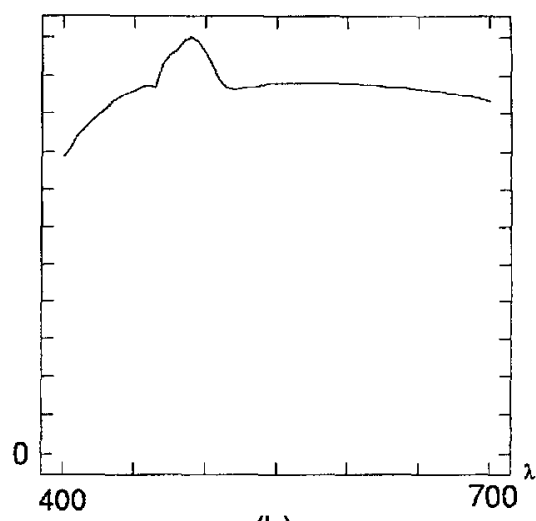

(b)

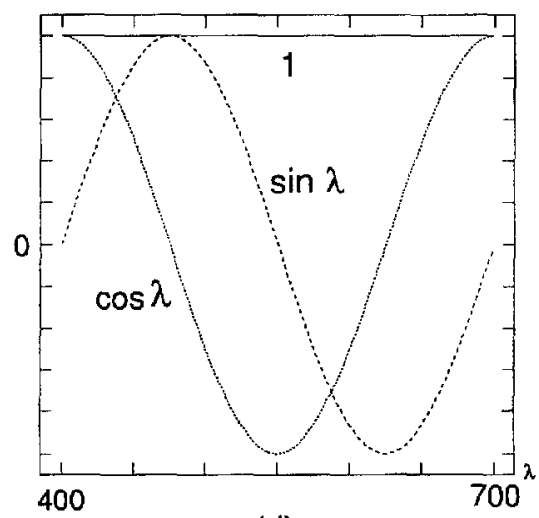

(d)

Figure 2. Spectral responses of (a) IR filter and CCD sensor, (b) lens, (c) color filters, and (d) Fourier basis functions.

where $S_{i}(\lambda)$ and $E_{j}(\lambda)$ are the basis functions for $s(\lambda)$ and $e(\lambda)$, respectively, and $\sigma_{i}$ and $\varepsilon_{j}$ are the scalar weighting factors. The relationship between the sensor response, illumination and surface reflection is given as (Maloney and Wandell, 1986)

$$
\underline{\mathbf{q}}=\underline{\Lambda^{\underline{\varepsilon}}} \underline{\sigma}, \quad \text { or } \quad \underline{\sigma}=\underline{\mathbf{V}}^{\underline{\varepsilon}} \underline{\mathbf{q}},
$$

where $\underline{\varepsilon}=\left[\varepsilon_{0}, \varepsilon_{1}, \varepsilon_{2}\right]^{T}, \underline{\sigma}=\left[\sigma_{0}, \sigma_{1}, \sigma_{2}\right]^{T}, \underline{\mathbf{V}}^{\underline{\varepsilon}}=\underline{\Lambda}^{\underline{\varepsilon}-1}$, and the elements of $\underline{\Lambda}^{\varepsilon}$ in the $k$ th row and $i$ th column are given by

$$
\Lambda_{k i}^{\varepsilon}=\int_{\lambda_{1}}^{\lambda_{2}} \sum_{j=0}^{2} \varepsilon_{j} E_{j}(\lambda) S_{i}(\lambda) Q_{k}(\lambda) d \lambda .
$$

The reflection vector $\underline{\sigma}$ is obtainable from the measurement $\mathbf{q}$ only when the three-dimensional approximation of the illumination $\underline{\varepsilon}$ is known. The process of finding the illumination color and discounting it is called color constancy. Computationally, the color constancy problem is to solve Eq. (9) for $\underline{\varepsilon}$ and $\sigma$ with the measurement q (see (Maloney, 1985; Maloney and Wandell, 1986) for detailed discussions). While the general solution for color constancy demands complex algorithms and some spectral and/or spatial constraints (Bajcsy et al., 1989; Buchsbaum, 1980; Forsyth, 1988; Maloney and Wandell, 1986; Wandell, 1987) it is simple to remove the known scene illumination measured with a reference object of known reflectance, and therefore it is practical for use. We use a reference plate with known reflectance to estimate $\underline{\varepsilon}$, and use the estimated sccne illumination $\underline{\varepsilon}$ for obtaining $\underline{\sigma}$ 's of objects in a scene. In this paper, this process is called "illumination neutralization" or "whitening" in contrast to the general color constancy in human and computer vision. This process can be used only when the objects of interest are under singly colored illumination, and a reference 
object can be applied as an active probe. We used the Fourier basis functions for $S_{i}(\lambda)$ and $E_{j}(\lambda)$ :

$$
\begin{aligned}
& S_{0}(\lambda)=E_{0}(\lambda)=1, \\
& S_{1}(\lambda)=E_{1}(\lambda)=\sin \lambda, \\
& S_{2}(\lambda)=E_{2}(\lambda)=\cos \lambda
\end{aligned}
$$

These functions are shown in Fig. 2(d) with the wavelength modified by letting $2 \pi(\lambda-400) / 300 \rightarrow \lambda$.

\section{Spectral Scene Radiance Model}

In order to develop a model to explain various optical phenomena such as shading, highlight, luster, shadow and inter-reflection, we use a three-dimensional spectral space constructed from three orthogonal basis functions. In the spectral space, called $\mathbf{S}$ space, we analyze three-dimensional color histograms formed by various surface reflections. The distribution of scene radiance in the $\mathbf{S}$ space is determined by spectral properties of surface reflectances and illumination, and is weakly influenced by scene geometry. In general, geometric information is collapsed in the $\mathbf{S}$ space. In this section, we show how the color clusters from body reflections and interface reflections appear in the $\mathbf{S}$ space. We also show how inter-reflections and shadows influence the color clusters of the body and interface reflections.

\subsection{S Space}

The $\mathbf{S}$ space is a color space based on three orthogonal basis functions $\left(S_{0}, S_{1}, S_{2}\right)$, and a color measurement in the $\mathbf{S}$ space is represented by a threc-dimensional vector $\underline{\gamma}$. Two basis functions are orthogonal if

$$
\int_{\lambda} S_{i}(\lambda) S_{j}(\lambda) d \lambda=0 .
$$

A set of three orthogonal basis functions is chosen so that the direction of the $S_{0}(\lambda)$-axis can be the direction of the neutral spectrum in the visible range of light, i.e., white or gray. The set of the first three Fourier bases is an example. The neutral direction is important in our model since it represents the spectral direction of neutral illumination and that of interface reflectance.

Certainly the Fourier bases may not be the best functions in terms of describing natural color. Other

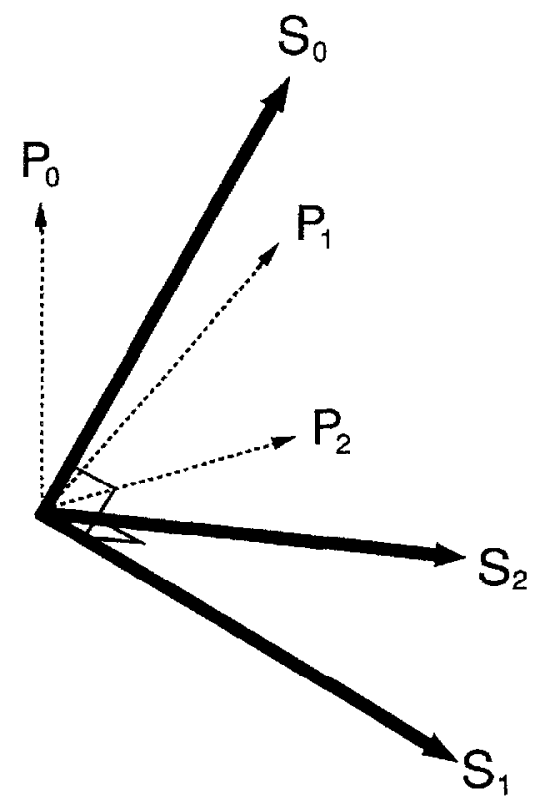

Figure 3. Construction of orthogonal spectral space.

non-orthogonal basis functions that may better describe natural colors can be used in constructing the orthogonal space through some modification. Figure 3 illustrates the construction of $\mathbf{S}$ space from a set of basis functions $\left(P_{0}, P_{1}, P_{2}\right)$. First the neutral direction for $S_{0}$ is determined such that it best approximates a flat spectrum in the least-squares sense in the visible range of light. Then two other vectors orthogonal to $S_{0}$ can be determined through Gram-Schmidt orthogonalization. It should be noted that while determination of $S_{0}$ is unique, $S_{1}$ and $S_{2}$ are not uniquely determined. If basis functions are orthogonal to each other but not oriented to the neutral direction, the modification is just a matter of rotation. The $\mathbf{S}$ space is a linear space in terms of scalar scaling and vector addition.

The two-dimensional space based on the normalized color vector $\hat{\gamma}=\left[\hat{\gamma_{1}}, \hat{\gamma_{2}}\right]^{T}=\left[\gamma_{1} / \gamma_{0}, \gamma_{2} / \gamma_{0}\right]^{T}$ is also useful for analysis and called $\hat{\mathbf{S}}$ space. The $\hat{\mathbf{S}}$ space is a chromaticity plane, and similar to the $1931 \mathrm{CIE}$ chromaticity plane and the normalized color space used by Healey and Binford (1988a) and Wyszecki and Stiles (1967). The $\mathbf{S}$ spacc and $\hat{\mathbf{S}}$ space are shown in Fig. 4(a) and (b), respectively.

The $\mathbf{S}$ space is similar to the opponent space which is convenient for describing brightness, hue and saturation. Hue is the perceptual attribute that allows colors to be classified as red, yellow, green, blue, and intermediates of these. Saturation is the attribute that 


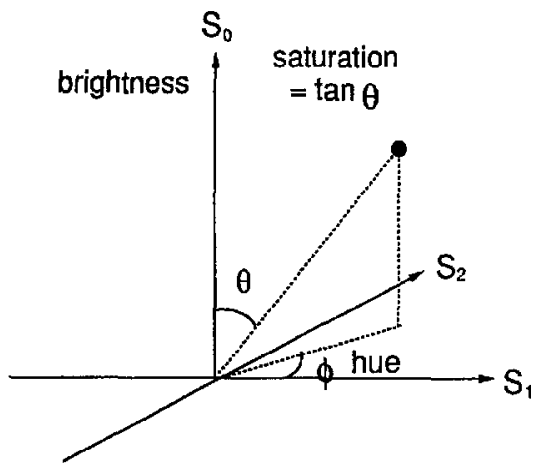

(a)

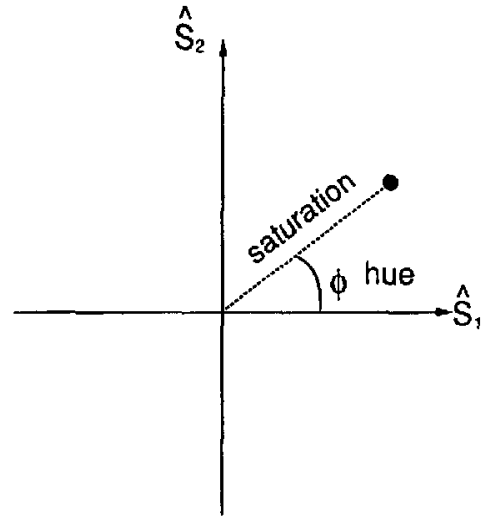

(b)

Figure 4. (a) $\mathbf{S}$ space, (b) $\hat{\mathbf{S}}$ space.

determines the degree of difference of a color from a gray of the same brightness (Beck, 1967; Wyszecki and Stiles, 1967). For analyzing reflections in the $\mathbf{S}$ and $\hat{\mathbf{S}}$, we define brightness, hue and saturation using the three-dimensional approximation of scene radiance $\underline{\gamma}=\left[\gamma_{0}, \gamma_{1}, \gamma_{2}\right]^{T}$ as (D'Zmura and Lennie, 1986).

$$
\begin{aligned}
& \text { brightness }=\gamma_{0} \text {, } \\
& \text { hue } \quad=\tan ^{-1}\left(\gamma_{2} / \gamma_{1}\right)=\tan ^{-1}\left(\hat{\gamma}_{2} / \hat{\gamma}_{1}\right) \text {, } \\
& \text { saturation }=\sqrt{\left(\gamma_{1}^{2}+\gamma_{2}^{2}\right)} / \gamma_{0}=\sqrt{\left(\hat{\gamma}_{1}^{2}+\hat{\gamma}_{2}^{2}\right)} \text {. }
\end{aligned}
$$

It should be noted that the saturation defined above is equivalent to the chroma in the psychophysical definitions of (Optical Society of America, 1953). In the $\mathbf{S}$ space, the hue is the phase angle and the saturation is the slope of the line from the color point to the origin as shown in Fig. 4. In the $\hat{\mathbf{S}}$ space, the hue and saturation are the polar coordinate values.

With the three-dimensional approximation, the SPD of a measured color spectrum is represented as a curve with only one peak in the visible range. Even when there is more than one distinct peak in a natural spectrum, a three-dimensional approximation with three filters results in only a smoothed version with one dominant peak (Maloney, 1986). Physically the brightness is the total spectral power of a color spectrum in the visible range. The hue is related to the wavelength of the spectral peak, and the saturation is the ratio of the spectrally varying component to the total power. With zero saturation, the spectral curve is flat, which means a neutral color.

\subsection{Body Reflection}

In the $\mathbf{S}$ space, the color clusters generated by shaded body reflections in general have arbitrary shape under multiply colored illumination. Under singly colored illumination, however, the clusters in the $\mathbf{S}$ space form a linear cluster. From Eqs. 1, 2 and 5 the sensor inputs are given by

$$
\left[\begin{array}{l}
q_{0} \\
q_{1} \\
q_{2}
\end{array}\right]=G_{B}\left[\begin{array}{l}
\int_{\lambda} e(\lambda) \rho_{B}(\lambda) Q_{0}(\lambda) d \lambda \\
\int_{\lambda} e(\lambda) \rho_{B}(\lambda) Q_{1}(\lambda) d \lambda \\
\int_{\lambda} e(\lambda) \rho_{B}(\lambda) Q_{2}(\lambda) d \lambda
\end{array}\right]
$$

Shading results from the variation in the geometric factor $G_{B}$ which is determined by surface orientation with respect to illumination direction. When $e(\lambda)$ and $\rho_{B}(\lambda)$ are constant over the differently shaded surfaces, the ratios of $q_{k}$ 's such as $q_{1} / q_{0}$ and $q_{2} / q_{0}$ are independent of $G_{B}$. Figure 5(b) shows the scaling of scene radiance $L_{r}(\lambda)$ when $e(\lambda)$ and $\rho_{B}(\lambda)$ are constant. Since the ratios of $q_{k}$ 's are constant over differently shaded surfaces, $\mathbf{q}$ 's from differently shaded surfaces form a linear cluster in the three-dimensional sensor space. This property has been previously suggested and used for color image segmentation ( Shafer, 1985; Shafer et al., 1990).

Since the body reflection $\underline{\gamma}_{B}$ is the linear transfor mation of the sensor inputs $q$, the ratios of $\gamma_{B_{i}}$ 's are also constant over the differently shaded surfaces. Therefore all the $\underline{\gamma}_{B}$ 's are collinear and they form a linear cluster in the $\boldsymbol{S}^{-\beta}$ space as shown in Fig. 5(c). Since the linear cluster is pointed toward the origin, the hue and saturation values of the $\underline{\gamma}_{B}$ 's in the linear cluster are 


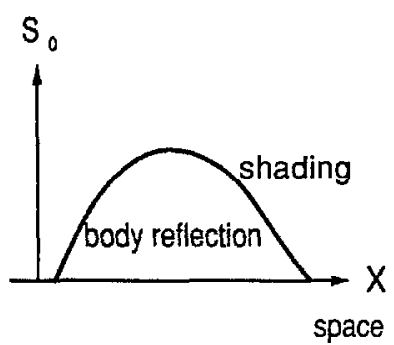

(a)

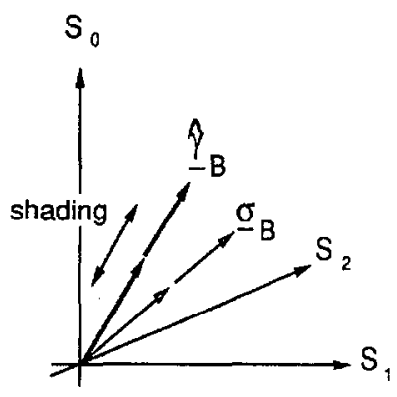

(c)

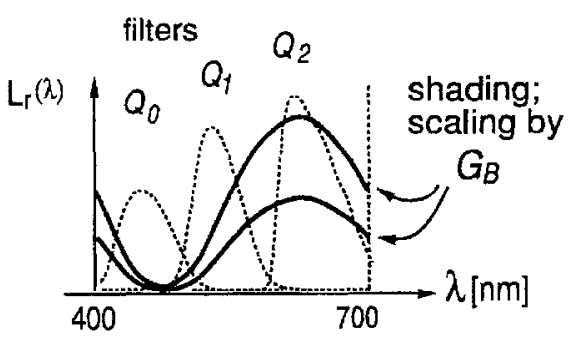

(b)

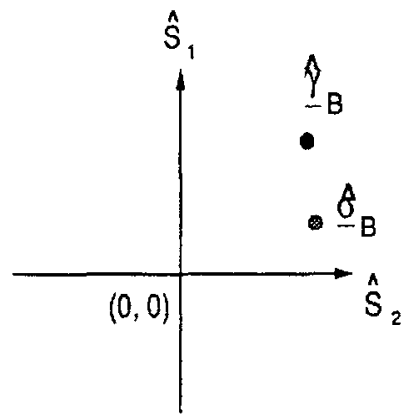

(d)

Figure 5. Shaded body reflection: (a) spatial distribution, (b) spectral curves, (c) in $\mathbf{S}$ space, (d) in $\hat{\mathbf{S}}$ space.

the same, and the linear cluster corresponds to a point cluster in the $\hat{\mathbf{S}}$ space as shown in Fig. 5(d). The orientation of the vector $\underline{\gamma}_{B}$ is determined by the body reflectance and illumination, and independent of geometry. When the illumination is neutral, $\underline{\gamma}_{B}$ becomes $\underline{\sigma}_{B}$ which is the surface reflection vector. It may be noted that when the variation of shaded body reflections is small, the linear cluster in the $\mathbf{S}$ space becomes a point cluster. Examples of this case include a planar geometry illuminated by a distant point source and any general objects under a hemispherically uniform source (Horn, 1986).

We show some simulations of shaded body reflections from a spherical object. The geometry shown in Fig. 6 applies to all the simulations shown in this section (Figs. 7, 8, 10-13). The coordinates $\left(X_{w}, Y_{w}, Z_{w}\right)$ denote the object coordinates, and the directions $\left(\theta_{V}, \phi_{V}\right)$ and $\left(\theta_{I}, \phi_{I}\right)$ denote the viewing direction and the illumination direction, respectively. We used the Fourier basis functions shown in Fig. 2(d), and SPD's measured from a real surface reflectance and incandescent light. Figure 7 shows a simulated image of a sphere, its color clusters in the $\mathbf{S}$ space and in the $\hat{\mathbf{S}}$ for $\left(\theta_{V}, \phi_{V}\right)$ $=\left(35^{\circ}, 0^{\circ}\right)$ and $\left(\theta_{I}, \phi_{I}\right)=\left(0^{\circ}, 0^{\circ}\right)$. The two separate linear clusters are shown together in the $\mathbf{S}$ space for two different illumination sources: neutral light and incandescent light. The different orientations of the linear

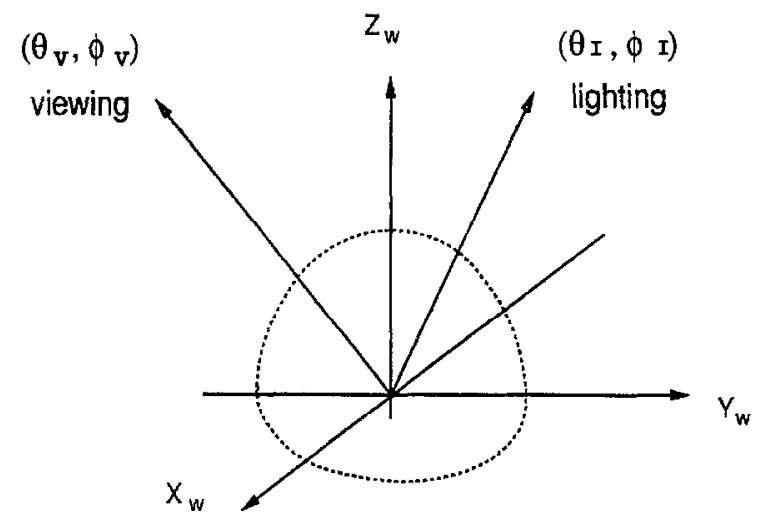

Figure 6. Coordinates for simulation.

clusters result from the different illumination colors. In the $\hat{\mathbf{S}}$ space, two point clusters are shown. Note that only one $S_{0}$-image is shown in Fig. 7 since the $S_{0}$ images for the neutral light and the incandescent light look the same except for different scaling.

When the two light sources are used together in a scene in different angles, the assumption of single illumination color does not hold. Figure 8 shows the same sphere at the same viewing angle but with the illumination angle $\left(\theta_{I 1}, \phi_{I 1}\right)=\left(0^{\circ}, 0^{\circ}\right)$ for the neutral light and $\left(\theta_{12}, \phi_{I 2}\right)=\left(45^{\circ}, 30^{\circ}\right)$ for the incandescent light. In the $\mathbf{S}$ space the color points form a planar 

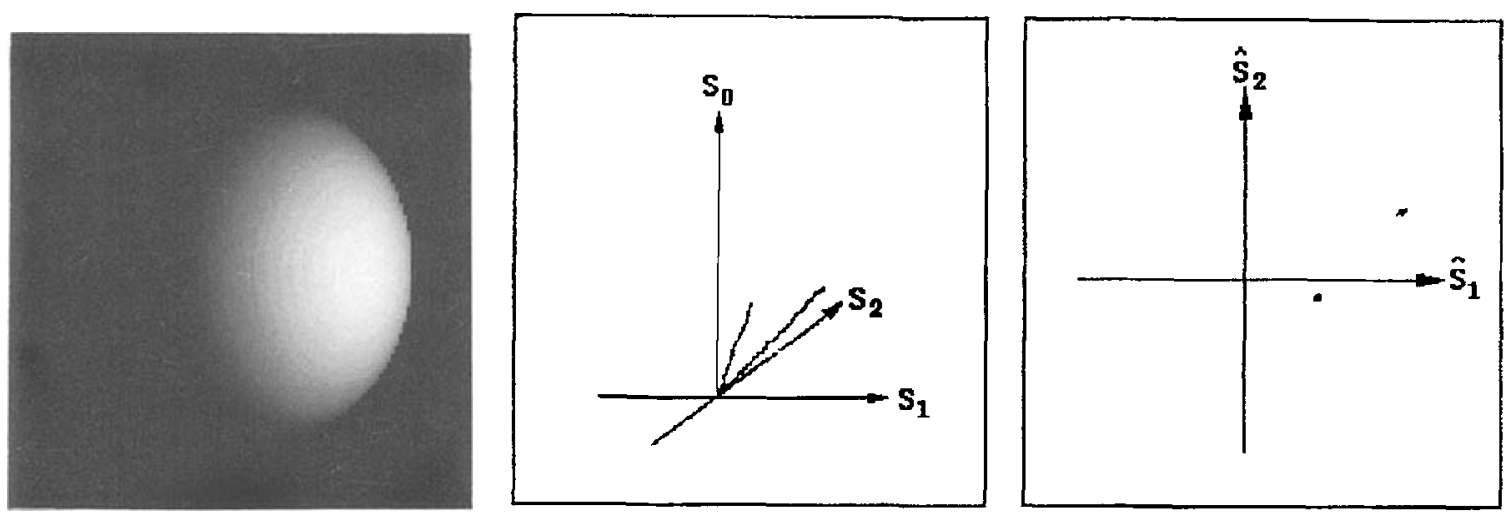

Figure 7. Shaded body reflection and two different color clusters under neutral light and under incandescent light in $\mathbf{S}$ space and $\hat{\mathbf{S}}$ space.
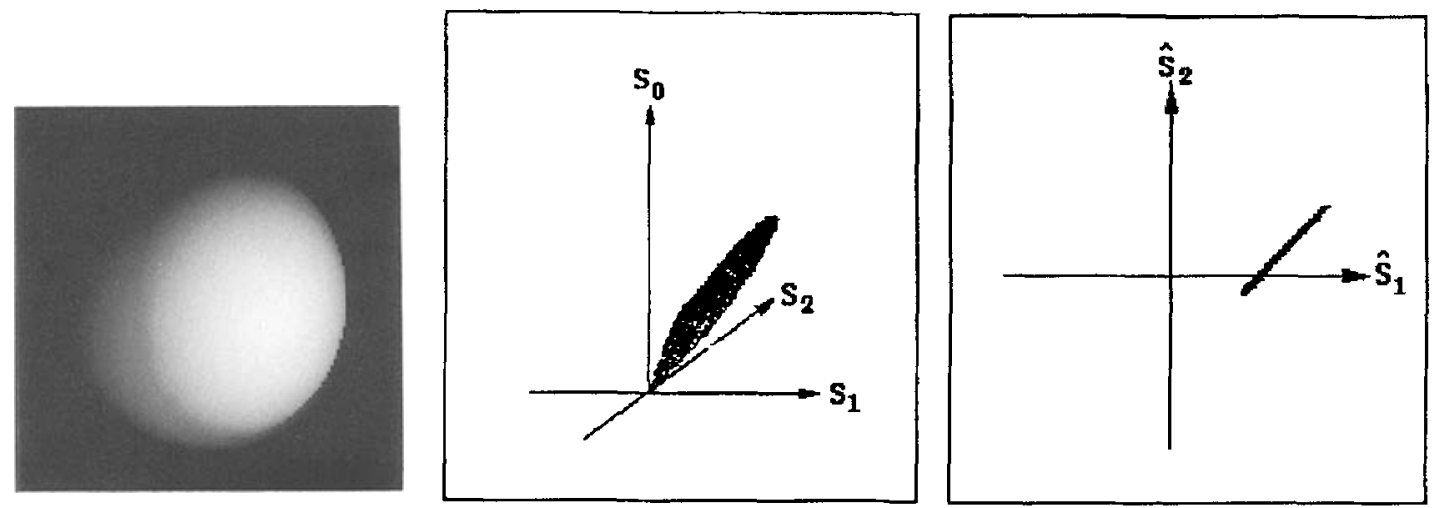

Figure 8. Body reflection under multiply colored illumination.

cluster since they are the linear combinations of the two body reflection lines, and in $\hat{\mathbf{S}}$ space, they form a linear cluster since they are the linear combinations of the two body reflection points. When there are more than two light sources from different angles, however, body reflections in general form a three-dimensional volume cluster in the $\mathbf{S}$ space and a planar cluster in the $\hat{\mathbf{S}}$ space.

\subsection{Interface Reflection}

From Eqs. 1, 2 and 5 the sensor inputs from interface reflections alone, e.g., from black dielectrics, are given by

$$
\left[\begin{array}{l}
q_{0} \\
q_{1} \\
q_{2}
\end{array}\right]=G_{S}\left(\theta_{r}, \phi_{r}\right)\left[\begin{array}{l}
\int_{\lambda} e(\lambda) \rho_{S}(\lambda) Q_{0}(\lambda) d \lambda \\
\int_{\lambda} e(\lambda) \rho_{S}(\lambda) Q_{1}(\lambda) d \lambda \\
\int_{\lambda} e(\lambda) \rho_{S}(\lambda) Q_{2}(\lambda) d \lambda
\end{array}\right]
$$

When $e(\lambda)$ and $\rho_{S}(\lambda)$ are constant in a region of interface reflections, the ratios of $q_{k}$ 's are independent of
$G_{S}\left(\theta_{r}, \phi_{r}\right)$. Like $\underline{\gamma}_{B}$ 's from body reflections, therefore, $\underline{\gamma}_{S}$ 's from interface reflections alone form a linear cluster in the $\mathbf{S}$ space. Because of the neutral reflectance, the direction of the linear cluster is the same as the illumination direction in the $\mathbf{S}$ space.

When interface reflections are mixed with underlying body reflections, they form a planar cluster and the shape of the cluster is determined by surface roughness and illumination geometry. If illumination is collimated and the surface is smooth, the interface reflections are specular and thus are spatially localized only in a small range of body reflection shading. In the $\mathbf{S}$ space, the interface reflections are added to a small part of a linear body reflection cluster. In this case, the interface reflections in the $\mathbf{S}$ space form a linear cluster connected to that of the underlying body reflections, and the two color clusters form a skewed $T$ or $L$ shape as suggested by Shafer (1985). If a surface is rough or illumination is extended, however, the interface reflections are diffuse, and they can be observed spatially over some area of the shaded body reflections as shown 


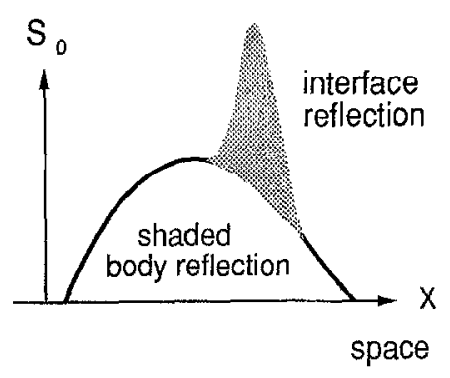

(a)

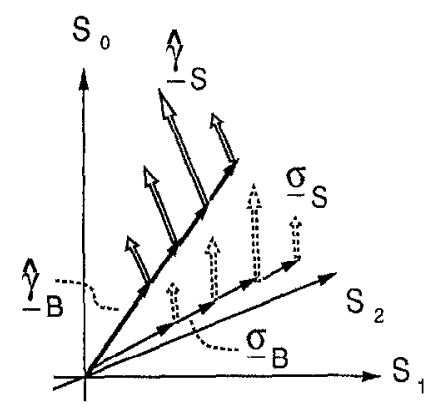

(c)

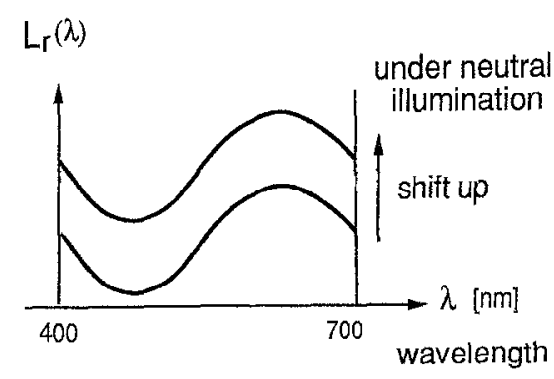

(b)

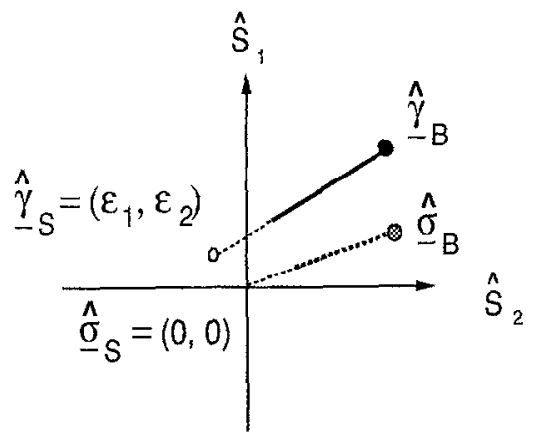

(d)

Figure 9. Interface reflection: (a) spatial distribution, (b) spectral curves, (c) in $\mathbf{S}$ space, (d) in $\hat{\mathbf{S}}$ space.

in Fig. 9(a). In the $\mathbf{S}$ space, therefore, the interface reflections are added to some differently shaded body reflections, and they form a planar cluster as shown in Fig. 9(c). The planar interface reflection cluster is coplanar with the linear body reflection cluster, and the two clusters in general form a skewed $P$ shape.

The orientation of the planar cluster is determined by the illumination color. When a neutral interface reflection under neutral illumination is added to a body reflection, it simply shifts up the spectral curve of the body reflection in the visible range of light as shown in Fig. $9($ b). In the $\mathbf{S}$ space, the addition of interface reflection shifts the body reflection upward along the $S_{0}$-direction as shown in Fig. 9(c). Under neutral illumination, therefore, a planar cluster formed by interface reflections is perpendicular to the $S_{1}-S_{2}$ plane, and coplanar with the $S_{0}$-axis. In the $\hat{\mathbf{S}}$ space shown in Fig. 9(d), a linear cluster is formed between the body reflection and the illumination $\left(\varepsilon_{1}, \varepsilon_{2}\right)$, which is also the chromaticity of the interface reflection according to the NIR model. When the illumination is neutral, the linear cluster is pointed toward the origin.

It is important to note that if an interface reflection is added to a body reflection under neutral light, the saturation of the combined reflection always decreases compared to that of the body reflection. This is because the addition of neutral interface reflection increases $\gamma_{0}$ in Eq. 13 without changing $\gamma_{1}$ and $\gamma_{2}$. On the other hand, hue is defined only in terms of $\gamma_{1}$ and $\gamma_{2}$ and thus is unaffected by the addition of interface reflection.

Figures 10,11 and 12 show the color clusters of interface and body reflections from a simulated sphere for different surface roughness and collimation of neutral illumination. We used the Torrance-Sparrow model for generating interface reflections from rough surfaces (Torrance and Sparrow, 1967). As shown in the figures, the color clusters in the $\mathbf{S}$ space are planar, and in general have skewed $P$ shapes in the simulations. This planarity is confirmed with the experimental data with real objects presented in Section 5. Depending on the views, surface roughness, and illumination collimation, there are some skewed $T$ - or $L$-shaped clusters that are special cases of the skewed $P$ shape. Figure 13 shows the the same sphere as in Figs. 10, 11 and 12 under multiply colored illumination: neutral and incandescent light sources from different directions. Like body reflections, interface reflections form a volume 

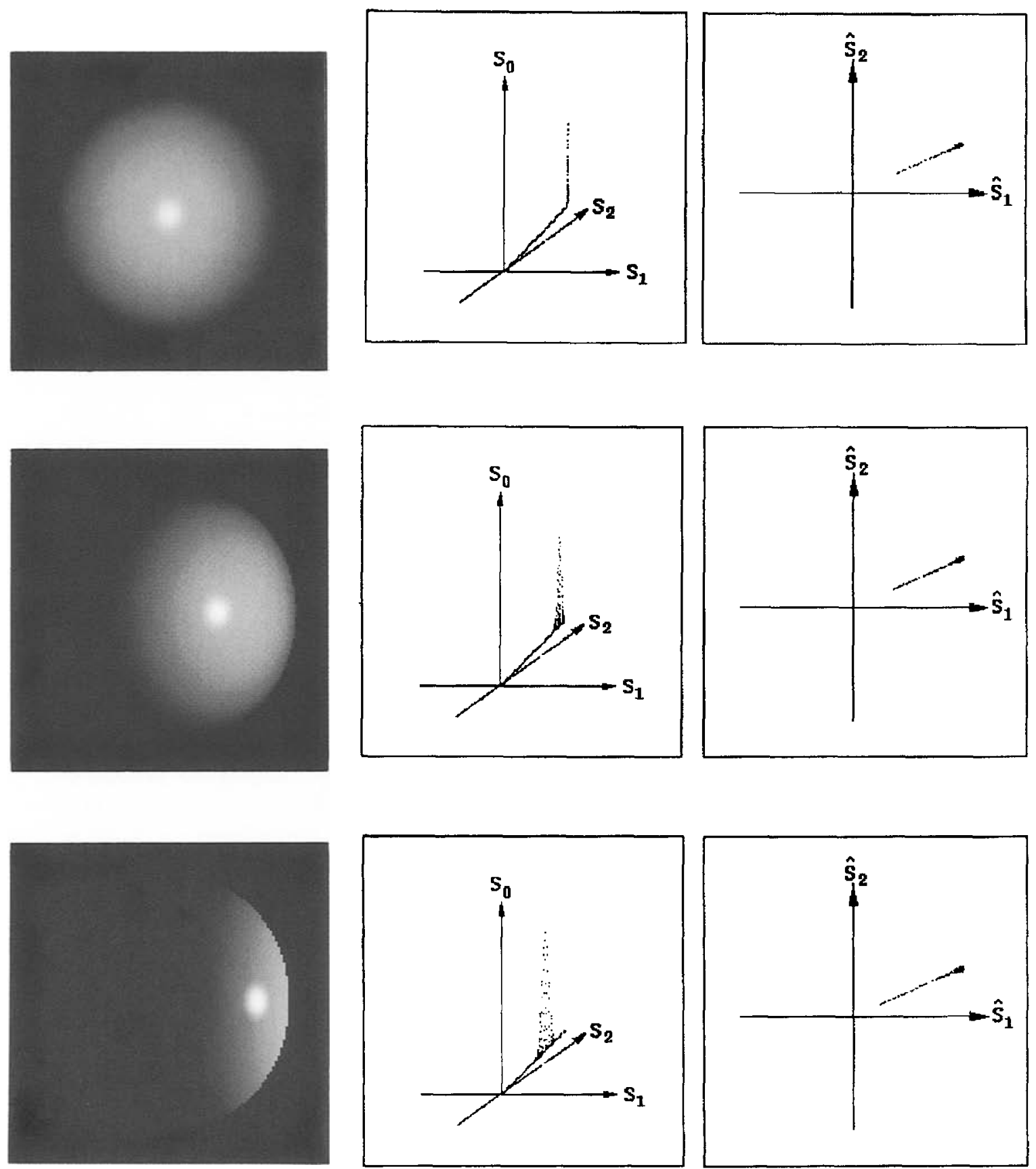

Figure 10. Reflections from smooth surface under collimated illumination for $\left(\theta_{I}, \phi_{I}\right)=\left(0^{\circ}, 0^{\circ}\right)\left(\theta_{V}, \phi_{V}\right)=\left(0^{\circ}, 0^{\circ}\right),\left(35^{\circ}, 0^{\circ}\right),\left(70^{\circ}, 0^{\circ}\right)$ and relative surface roughness $=0.1$. 

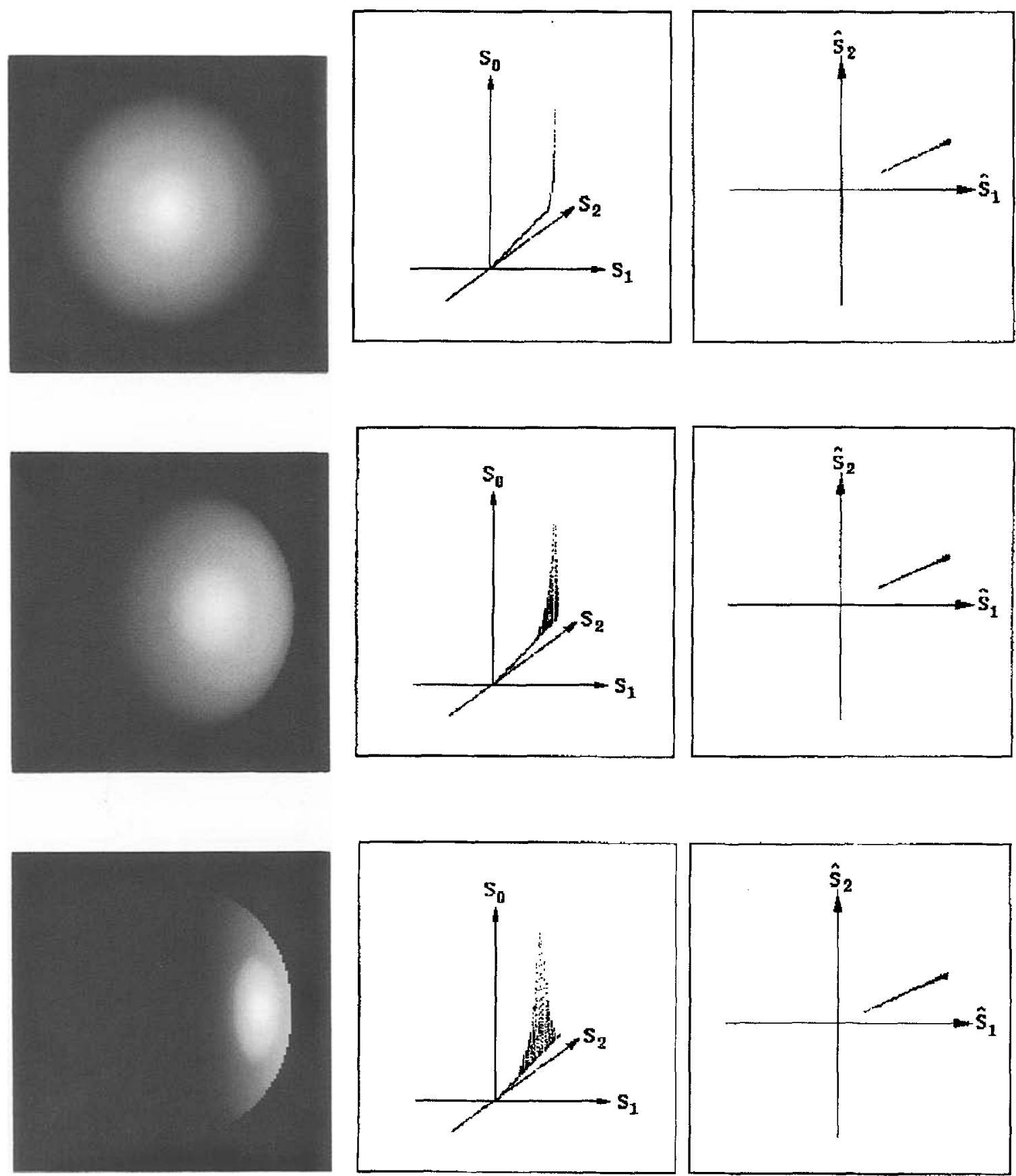

Figure 11. Reflections from smooth surface under extended illumination for $0^{\circ}<\theta_{I}<30^{\circ}, 0^{\circ}<\phi_{I}<360^{\circ},\left(\theta_{V}, \phi_{V}\right)=\left(0^{\circ}, 0^{\circ}\right),\left(35^{\circ}, 0^{\circ}\right)$ : $\left(70^{\circ}, 0^{\circ}\right)$ and relative surface roughness $=0.1$. 

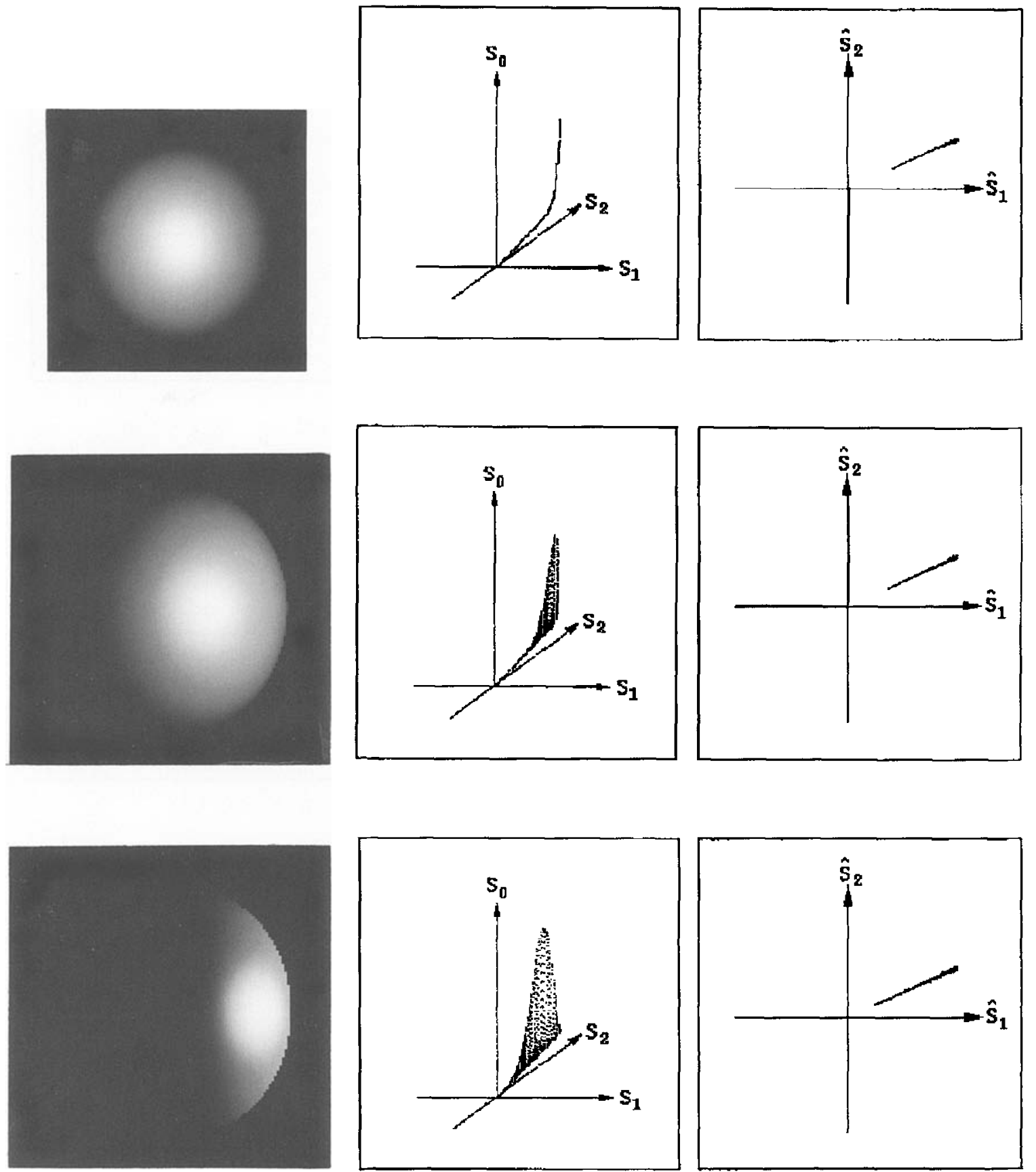

7igure 12. Reflections from rough surface under collimated illumination for $\left(\theta_{I}, \phi_{I}\right)=\left(0^{\circ}, 0^{\circ}\right)\left(\theta_{V}, \phi_{V}\right)=\left(0^{\circ}, 0^{\circ}\right),\left(35^{\circ}, 0^{\circ}\right),\left(70^{\circ}, 0^{\circ}\right)$ an elative surface roughness $=\mathbf{0 . 3}$. 

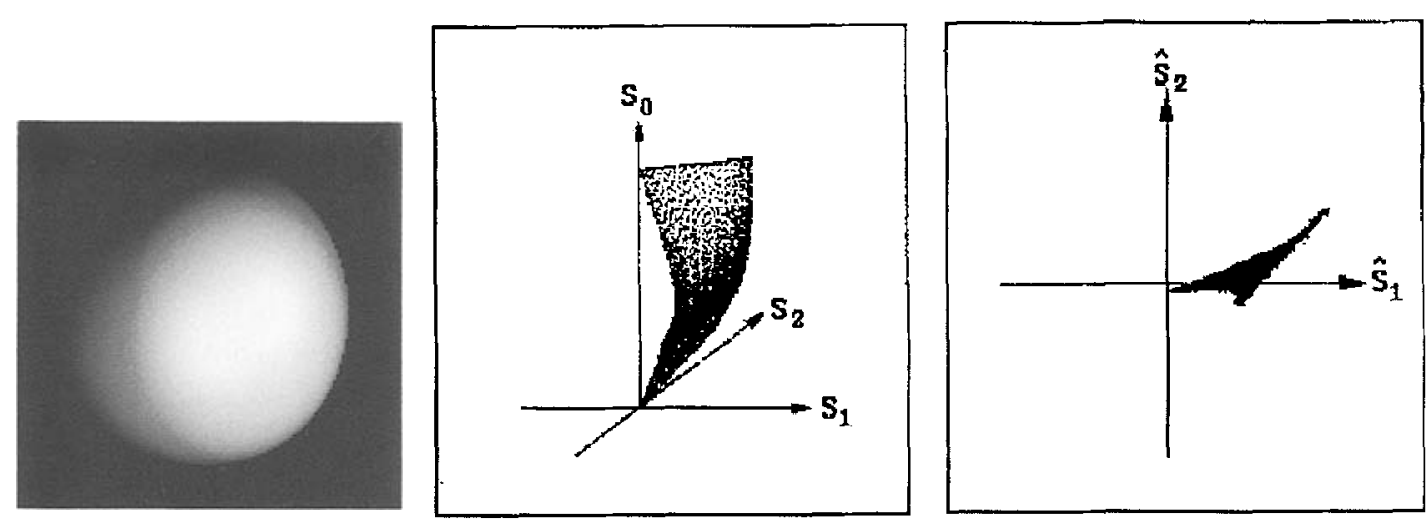

Figure 13. Reflections under multiply colored illumination for $\left(\theta_{I 1}, \phi_{I I}\right)=\left(0^{\circ}, 0^{\circ}\right)$ and $\left(\theta_{I 2}, \phi_{I 2}\right)=\left(45^{\circ}, 30^{\circ}\right)\left(\theta_{V}, \phi_{V}\right)=\left(0^{\circ}, 0^{\circ}\right)$, $\left(35^{\circ}, 0^{\circ}\right),\left(70^{\circ}, 0^{\circ}\right)$ and relative surface roughness $=0.3$.

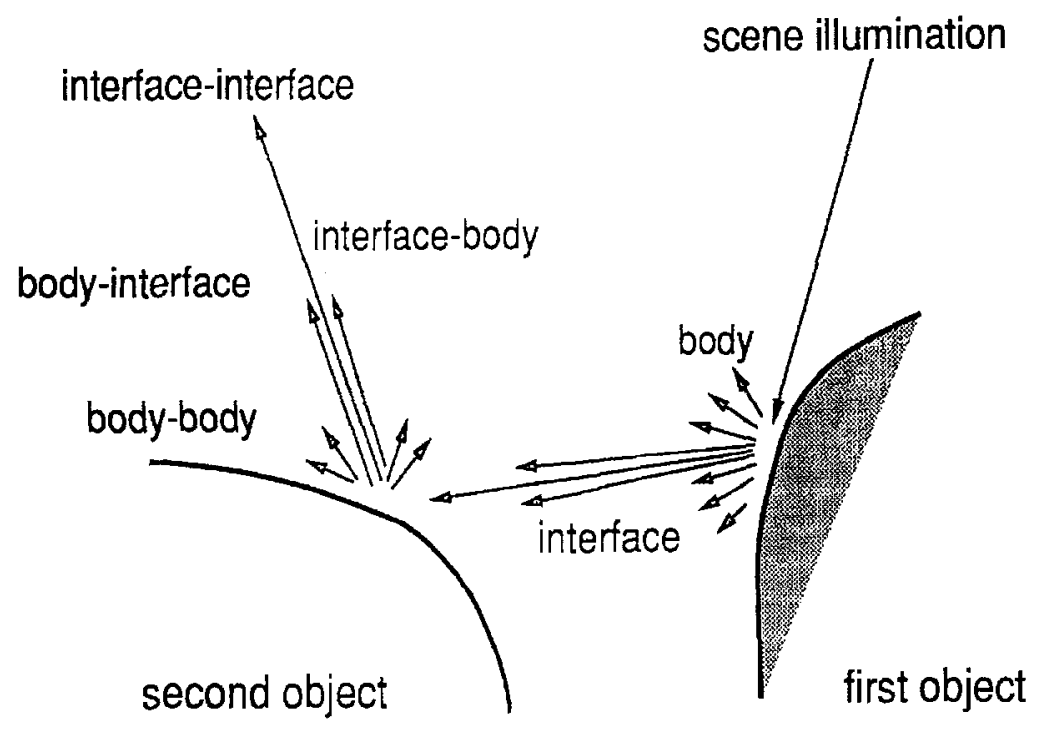

Figure 14. Inter-reflection.

cluster in general under multiply colored illumination in the $\mathbf{S}$ space, and a planar cluster in the $\hat{\mathbf{S}}$ space.

\subsection{Variation of Illumination by Multiple Objects}

We have so far explained the variation of spectral scene radiance from one object without considering optical interactions between object surfaces. Multiple objects or different parts of one object can result in local variation of illumination such as inter-reflections and shadows.

3.4.1. Inter-Reflection. When there are many objects in a scene, a surface patch of an object receives not only the light from the illumination sources but the reflected light from other objects. An object surface is a secondary source of light that radiates a part of the received light from primary sources, and it causes a local change of illumination as illustrated in Fig. 14. The reflection from more-than-one surfaces is called inter-reflection or mutual reflection.

Inter-reflections consist of components with both spectral and geometric interrelationships. Figure 14 shows four components involved in the inter-reflection betwcen two diclectric surfaces. The first reflection, has the interface and the body reflection components, and the second reflection in the other object has the four components: the interface-interface, the interfacebody, the body-interface and the body-body reflections. Object surfaces are secondary light sources which are generally extended depending on the object size. In 
indoor environments, reflections from walls and ceiling are the major sources of ambient light.

Inter-reflections can be analyzed in a simplified manner for two object surfaces as follows. The global illumination is assumed to be singly colored and the objects are uniform in hue and saturation. The two local illumination components from the first reflection are illustrated in Fig. 14(b). The scene radiance from inter-reflection is

$$
\begin{aligned}
L_{r}(\lambda)= & e(\lambda)\left[G_{S}^{\prime}\left(\theta_{i r}, \phi_{i r}\right) \rho_{S}^{\prime}(\lambda)+G_{B}^{\prime} \rho_{B}^{\prime}(\lambda)\right] \\
& \times\left[G_{S}\left(\theta_{r}, \phi_{r}\right) \rho_{S}(\lambda)+G_{B} \rho_{B}(\lambda)\right],
\end{aligned}
$$

where the first reflection is denoted by ', and $i r$ denotes the direction from the object of first reflection and to the patch where the second reflection occurs. The first reflection provides two spectral components of local illumination. The interface reflection component of the local illumination is determined by setting $G_{B}^{\prime} \rho_{B}^{\prime}(\lambda)$ to 0 in $\mathrm{Eq} .16$ as

$$
\begin{aligned}
L_{r}^{\text {first-interface }}(\lambda)= & e(\lambda) G_{S}^{\prime}\left(\theta_{i r}, \phi_{i r}\right) \mu_{S}^{\prime}(\lambda) \\
& \times\left[G_{S}\left(\theta_{r}, \phi_{r}\right) \rho_{S}(\lambda)+G_{B} \rho_{B}(\lambda)\right]
\end{aligned}
$$

When the first object is dielectric, the local illumination provided by the first interface reflection is the same as the global scene illumination since $\rho_{S}^{\prime}(\lambda)$ is spectrally neutral according to the NIR model. Therefore the first-interface (interfacc-interface and interface-body) inter-reflections are spectrally indistinguishable from direct reflections.
Under the local illumination due to the body reflection component of the first reflection, the scene radiance is

$$
\begin{aligned}
L_{r}^{\text {first-body }}(\lambda)= & e(\lambda) G_{B}^{\prime} \rho_{B}^{\prime}(\lambda) \\
& \times\left[G_{S}\left(\theta_{r}, \phi_{r}\right) \rho_{S}(\lambda)+G_{B} \rho_{B}(\lambda)\right] .
\end{aligned}
$$

If the body reflectance $\rho_{B}^{\prime}(\lambda)$ is colored, it provides a local change of illumination different from the global scene illumination. When only the global scene illumination color is discounted using a reference reflectance, the body reflection from the first object remains as a local variation of illumination that deviates from the neutralized illumination. Therefore the first-body (body-interface and body-body) interreflection can change the perceived hue as well as the saturation of a surface, and in the $\mathbf{S}$ space, the color pixels influenced by the first-body inter-reflection generally do not fall in the planar region formed by direct interface and body reflections. Strong first-body interreflections can form a completely different cluster from the planar cluster of direct reflections, but weak firstbody inter-reflections result in small deviations. When interface-interface reflections are involved in the interreflections, the saturation values are likely to decrease compared to those of the underlying body reflections.

3.4.2. Shadow. Shadows result from the obstruction of light from illumination sources by other objects as shown in Fig. 15(a). When the light sources are partially obstructed, the geometric weighting factors $G_{S}$ and $G_{B}$ decrease due to the obstruction of light from some illumination directions. The decrease in $G_{S}$ and
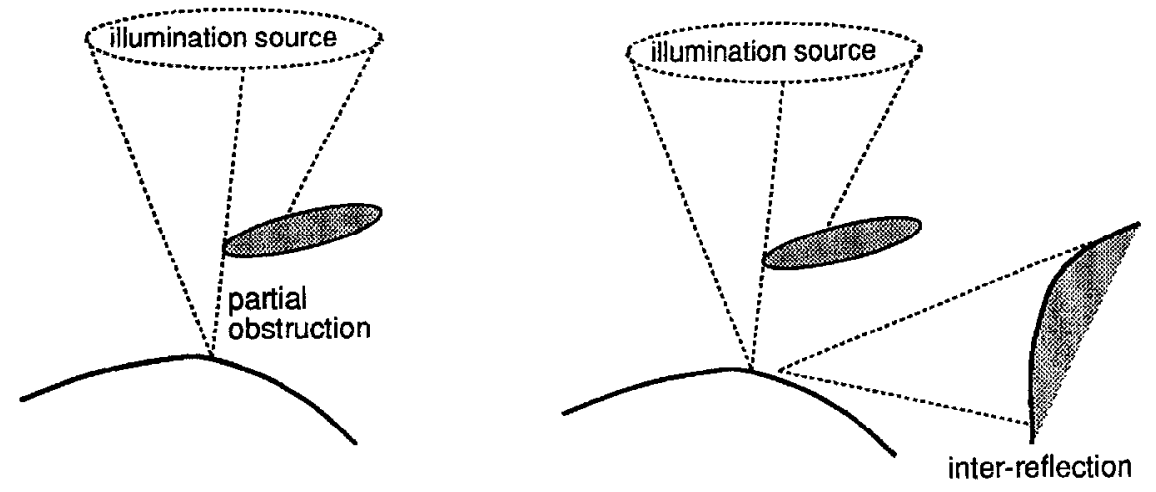

(a)

(b)

Figure 15. Shadow: (a) partial obstruction of illumination light, (b) local variation of illumination due to inter-reflection. 


\section{$S_{0}$}

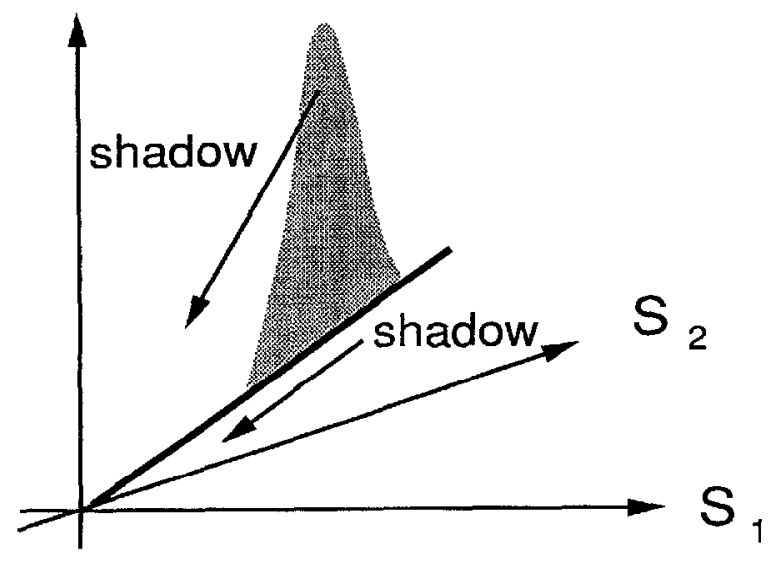

Figure 16. Scaling of color points by shadow.

$G_{B}$ results in the scaling of color points in the $\mathbf{S}$ space toward the origin as illustrated in Fig. 16, and therefore no spectral parameters such as hue and saturation are changed. A color cluster in the $\mathbf{S}$ space can change its shape due to a partial shadow since only the shadowed color points in the cluster are weighted toward the origin depending on the amount of light obstruction. If a surface patch is completely obstructed from all the illumination sources, the surface patch is not observable.

This shadow model is correct only under singly colored illumination. When there are many illumination sources of different SPD's, partial obstruction of light from some sources can change the spectral composition of illumination that a shadowed surface patch receives, thus the hue and saturation values can be affected. Under singly colored illumination, however, only sources of different illumination from the scene illumination are inter-reflections as illustrated in Fig. 15(b). Therefore the change in huc and saturation values in a shadowed area results only from the firstbody inter-reflections. For more discussions of color models for shadows, see (Funka-Lea and Bajcsy, 1993; Jepson et al., 1986).

\section{Color Image Segmentation}

We have analyzed the variation of surface reflections in the $\mathbf{S}$ and $\hat{\mathbf{S}}$ space, and interpreted it in terms of brightness, hue and saturation. For a surface with uniform body reflectance in hue and saturation under singly colored illumination, our scene radiance model is summarized as follows.

- Brightness changes when there are shaded body reflections, interface reflections, shadows or interreflections.

- Shading does not change the hue or the saturation of body reflections.

- Shadows do not change the hue or the saturation of body reflections when inter-reflections are not involved. Shadows decrease brightness.

- Interface reflections always decrease the saturation of colored body reflections, and increase the brightness.

- Inter-reflections result from the local change of illumination by the reflections from other objects.

- Interface-interface inter-reflections decrease the saturation as do the direct interface reflections.

- Interface-body inter-reflections are indistinguishable from the direct body reflections.

- Body-interface and body-body reflections in general cause a change in the hue and the saturation.

When the inter-reflections between neighboring dielectric objects include interface-interface interreflections, the saturation tends to decrease.

Based on this model, we propose an algorithm for detecting and separating interface reflections and some inter-reflections from body reflections using an image segmentation technique. The approach presented in this paper is to decompose three-dimensional color image data into a set of one-dimensional data (hue and saturation) and to use an existing image segmentation technique. For image segmentation, the following assumptions are required.

- Objects in a scene have uniform body reflectances (albedoes) in hue and saturation, but not in brightness. With such objects in a scene, a color image consists of piecewise-uniform patches in hue and saturation, i.e., the image is a Mondrian picture only in hue and saturation for body reflections, but not for interface or combined interface and body reffections.

- Global scene illumination is uniform in color whether extended or collimated, i.e., singly colored illumination.

Note that these assumptions are also required for the algorithms by Gershon (1987) and Shafer et al. (1990). 


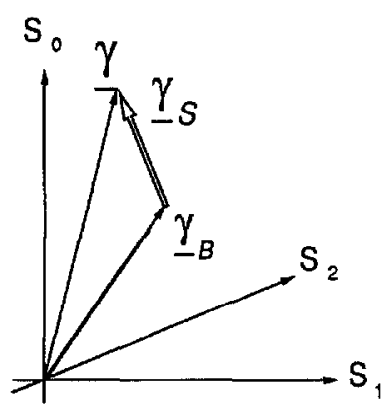

(a)

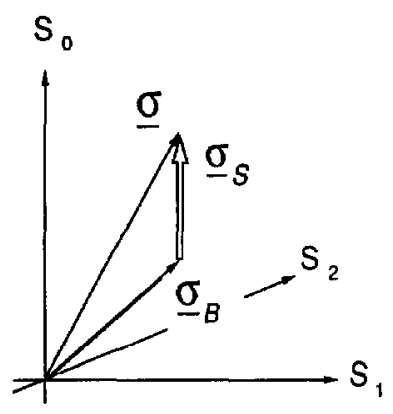

(c)

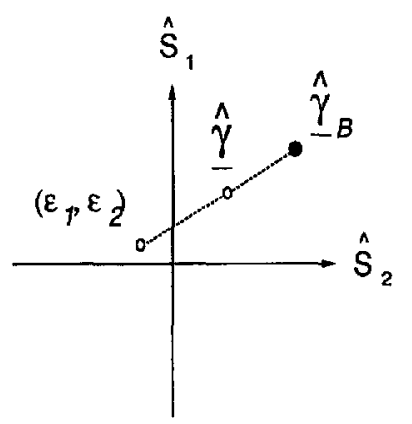

(b)

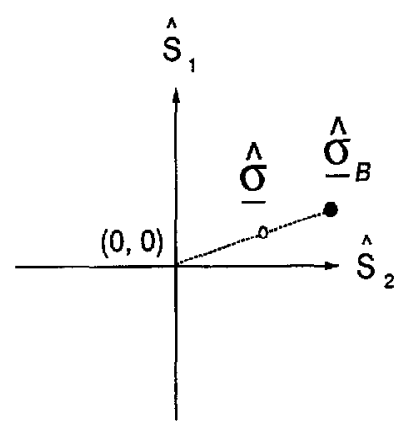

(d)

Figure 17. Addition of two reflection components: under colored illumination in (a) $\mathbf{S}$ space, (b) $\hat{\mathbf{S}}$ space, and under neutral illumination in (c) $\mathbf{S}$ space, (d) $\hat{\mathbf{S}}$ space.

\subsection{Separation of Interface and Body Reflections}

The problem of separating interface reflections from body reflections is to obtain $\underline{\gamma}_{S}$ and $\underline{\gamma}_{B}$ separately from the measurement $\underline{\gamma}=\underline{\gamma}_{S}+\underline{\gamma}_{B}$. Figure 17(a) illustrates the spectral vectors for the interface $\left(\underline{\gamma}_{S}\right)$ reflections, the body reflections $\left(\underline{\gamma}_{B}\right)$ and the total (combined interface and body) reflection $(\gamma)$. Without extra information, the problem of separation is generally underconstrained, and some constraints must be provided to solve the problem. For our algorithm presented in this paper, the constraints are given by the assumptions stated above and the known illumination color. The first constraint is provided by the estimated direction of the linear cluster by shaded body reflections. Under the assumptions of uniform reflectance and single illumination color, a color cluster from body reflections is linear in the $\mathbf{S}$ space, and segmentation of the body reflection cluster would provide the information about the direction of $\gamma_{B}$.

The above constraint is still insufficient for determining either the interface reflection vector $\underline{\gamma}_{S}$ or the magnitude of the body reflection vector $\underline{\gamma}_{B}$. The second constraint is provided by the known illumination direction, which is the same as the direction of an interface reflection vector for dielectrics according to the NIR model. With the second constraint, it is possible to determine $\underline{\gamma}_{S}$ and $\underline{\gamma}_{B}$ uniquely as shown in Fig. 17(a). The illumination color can be reliably estimated with a reference plate. Figure 17(b) shows the body reflection $\underline{\hat{\gamma}}_{B}$, the interface reflection $\hat{\hat{\gamma}}_{S}$ and the total reflection $\hat{\gamma}$ in the $\hat{S}$ space. Because of the NIR property, the interface reflection has the same chromaticity as that of the illumination $\left(\varepsilon_{1}, \varepsilon_{2}\right)$.

When illumination is neutralized, the color points in the $\mathbf{S}$ space are arranged as shown in Fig. 17(c). The interface reflection has the same direction as the $S_{0^{-}}$ axis which is perpendicular to the $S_{1}-S_{2}$ plane, and in the $\hat{\mathbf{S}}$ space, the origin $(0,0)$ is the interface reflection chromaticity as shown in Fig. 17(d). The advantage of illumination neutralization for image segmentation is that the hue values of interface and body reflections are the same, and the presence of interface reflections can be detected by reduced saturation compared to that 


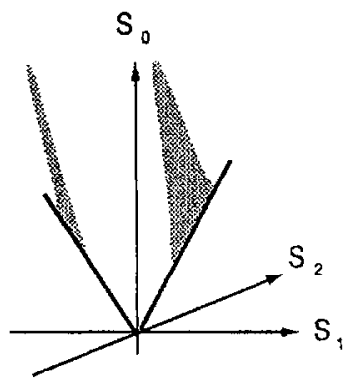

(a)

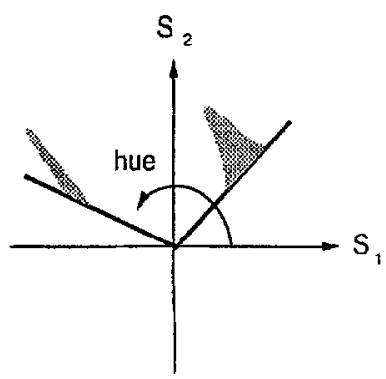

(b)

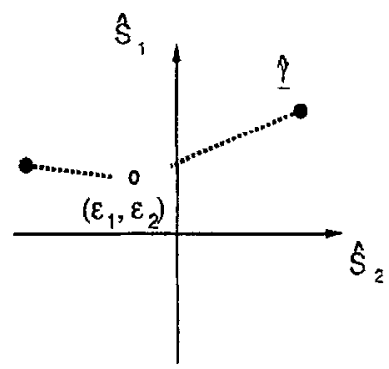

(c)

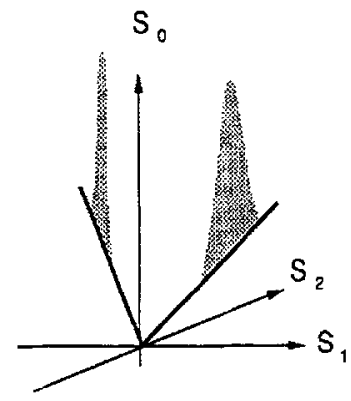

(d)

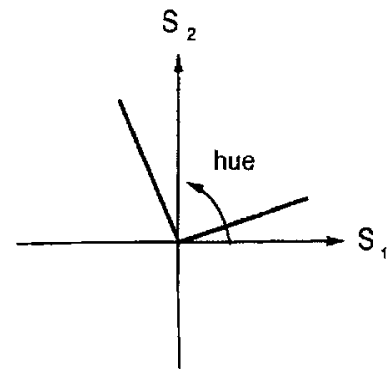

(e)

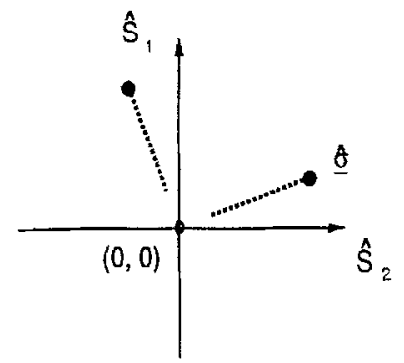

(f)

Figure 18. Color clusters under colored illumination: (a) $\mathbf{S}$ space, (b) top view of $\mathbf{S}$ space, (c) $\mathbf{S}$ space. Color clusters under neutral illumination: (c) $\mathbf{S}$ space, (d) top view of $\mathbf{S}$ space, (e) $\hat{\mathbf{S}}$ space.

of underlying body reflections. In the following, we discuss the details of hue and saturation segmentation.

\subsection{Segmentation by Hue}

Under the whitened illumination, the interface reflection clusters in the $\mathbf{S}$ space are all aligned with the brightness direction. As a result, the interface reflections have the same hue value as the underlying body reflection clusters. Figure 18(a) and (d) illustrate color clusters under colored and neutral illumination in the S space, respectively, and Fig. 18(b) and (e) are the top views of the clusters shown in Fig. 18(a) and (d), respectively. In Fig. 18(c) and (f), the normalized color clusters in the $\hat{\mathbf{S}}$ space are shown. Without illumination neutralization, the planar clusters are not generally parallel with the $S_{0}$-axis, but tilted depending on the illumination color. Therefore the interface reflections are dispersed in hue values depending on the illumination as shown in Fig. 18(b) and (c). Under neutral or neutralized illumination, the planes formed by the interface and body reflections are parallel with the $S_{0^{-}}$ axis and perpendicular to the $S_{1}-S_{2}$ plane as shown in Fig. 18(d). Therefore, the interface and the body reflections from the same object are aligned at the same 
hue under neutral illumination as shown in Fig. 18(e) and (f), and therefore they can be segmented together by hue segmentation.

For hue segmentation after illumination neutralization, histograming, region-growing or region-splitting algorithms can be used for segmenting regions of uniform hue values (Hanson and Riseman, 1978; Leonardis et al., 1990; Ohlander et al., 1978). Among others, we used a modified version of the algorithm proposed by Leonardis et al. (1990). The algorithm was developed originally for the surface segmentation of range data using variable-order (up to second order) bi-variate polynomials and using iterative regression. The modified version scarchcs only for regions of constant and uniform hue or saturation (zero-th order), and rejects any regions where hue or saturation values vary.

For hue segmentation, the algorithm places seeds of seven-by-seven-pixel windows randomly but evenly to cover a whole image, and simultaneously grows the seeds until they reach discontinuities or varying hue values. Therefore the growth process is limited only to regions of constant hue values, but the regions grown by each seed can overlap. If a seed is placed where hue values are not uniform, it is not allowed to grow and thus is eliminated. When the growth is finished, the algorithm merges the regions of similar hue values. The criterion of uniformity in growing and merging is set by a parameter, called the "uniformity parameter", which can be determined by the system noise and distortion. For hue segmentation, we use only the algorithm of seed growing and region merging as described above, but do not use hue histograms, though they may be used for confirming the result of hue segmentation. An example of the segmentation process with a color image of real objects is shown in Section 5.

\subsection{Segmentation by Saturation}

The color clusters in a hue-segmented region lie in a two-dimensional plane which intersects with the $S_{0^{-}}$ axis and is perpendicular to the $S_{1}-S_{2}$ plane in the $\mathbf{S}$ space. Figure 19(a) and (b) illustrate a planar cluster in the $\mathbf{S}$ space and a linear cluster in the $\hat{\mathbf{S}}$ space after hue segmentation, respectively. In each hue-segmented plane in the $\mathbf{S}$ space, body reflections form a linear cluster, and thus have a uniform saturation. On the other hand, saturation decreases where interface reflections are added. If the saturation value of body reflections is found, therefore, interface reflections can be detected by their decrease in saturation. For finding body reflections of uniform saturation, we use the algorithm of seed growing and region merging with a uniformity parameter determined by the system noise level. The segmented region of uniform hue and saturation represents the linear cluster of body reflections, and the regions of reduced saturation are those of interface reflections. Two assumptions required for this detection algorithm are as follows.

- The body reflections are visible in a hue-segmented region.

- If a hue-segmented region is totally covered with diffuse interface reflections, the body reflections are not visible and it is not possible to determine the saturation values of the linear body reflection clusters. Therefore, the result of the detection and separation is correct only when the body reflections are observable in a hue-segmented region.

- The linear clusters are formed only by body reflections.

- If the diffuse interface reflections have uniform saturation values in some very extended areas, they form an extra linear cluster in addition to that of body reflections. In this case the linear cluster formed by the diffuse interface reflection is segmented as a separate body reflection region.

Once the presence of an interface reflection is detected, the interface reflection vector is given by the spectral distance from the measured total reflection to the linear body reflection cluster along the neutral illumination direction (the $S_{0}$-direction) as shown Fig. 17(c).

When there is more than one object in the same hue plane, the simple method described above may not detect interface reflections due to possible spectral overlaps in the $\mathbf{S}$ space, and spatial information is necessary for segmentation. Figure 19(c) and (d) show color clusters from two objects $\alpha$ and $\beta$ with same hue in the $S$ and the $\hat{\mathbf{S}}$ space, respectively, and Fig. 19(e) shows a possible spatial distribution of the reflections. The symbols $\alpha_{B}$ and $\beta_{B}$ denote the color clusters from body reflections, and $\alpha_{S 1}, \alpha_{S 2}$ and $\beta_{S}$ denote the color clusters from interface reflections. In Fig. 19(c) and (d), $\alpha_{S 2}$ spectrally overlaps $\beta_{B}$ and $\beta_{S}$. When different color clusters overlap in the $\mathbf{S}$ space, ambiguities arise and they cannot be resolved only in the $\mathbf{S}$ space. Therefore spatial information in an image needs to be exploited. Below we explain an algorithm that uses spatial information with an example shown in Fig. 19(e).

The first step of the algorithm is to segment the body reflection regions $\alpha_{B}$ and $\beta_{B}$ by finding the regions of uniform saturation. The algorithm of seed growing and 


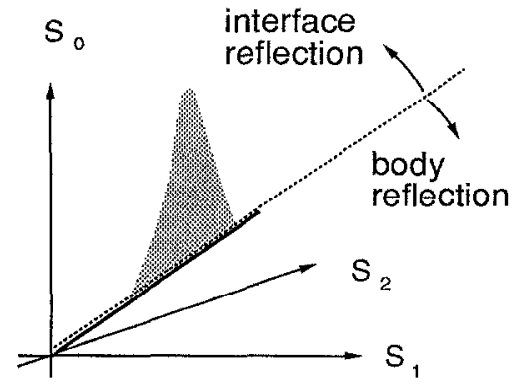

(a)

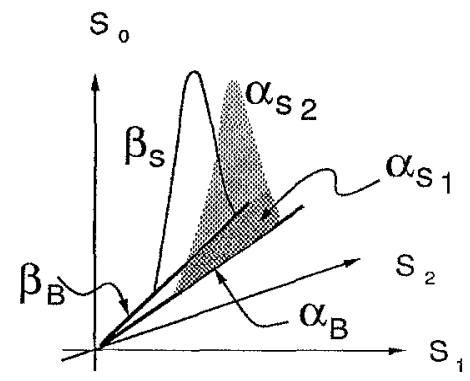

(c)

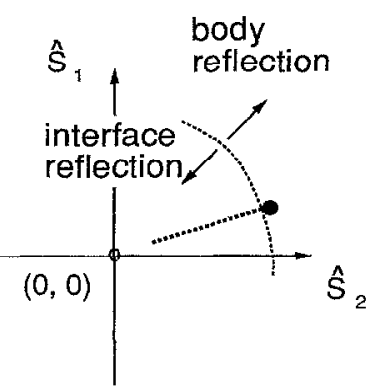

(b)

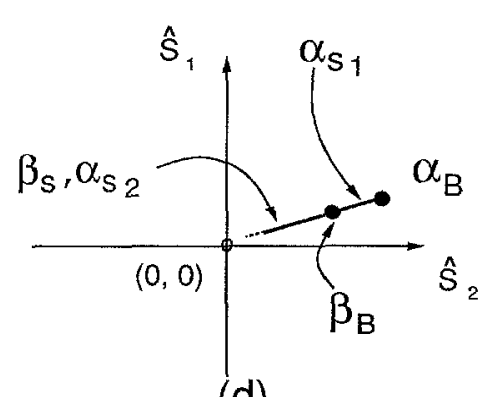

(d)

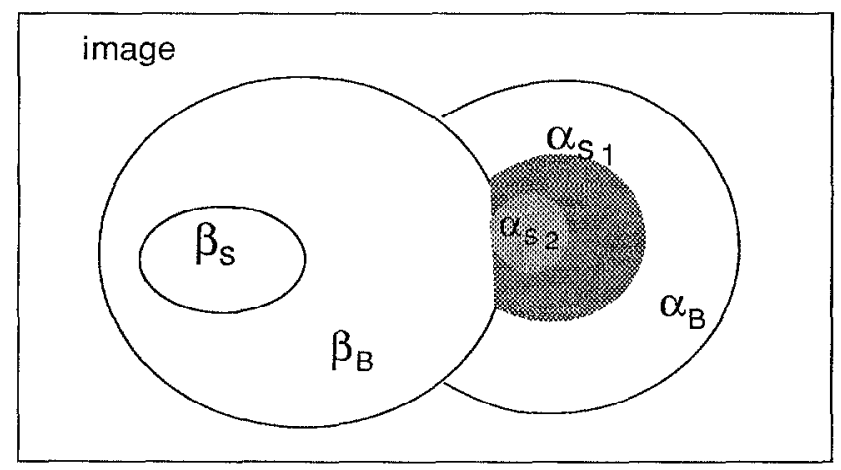

(e)

Figure 19. Color clusters after hue segmentation: (a) one object in $\mathbf{S}$ space, (b) one object in $\hat{\mathbf{S}}$ space, (c) two objects in $\mathbf{S}$ space, (d) two objects in $\hat{\mathbf{S}}$ space, (e) spatial distribution of two objects and reflections.

region merging searches for the patches of uniform saturation within a uniformity parameter, and rejects any regions with saturation variation beyond the uniformity parameter. In Fig. 19(e), the body reflection regions $\alpha_{B}$ and $\beta_{B}$ have uniform saturation values, and the interface reflection regions $\alpha_{S 1}, \alpha_{S 2}$ and $\beta_{S}$ are most likely to have variations in saturation values. The uniform saturation regions are used to find the most probable linear clusters of body reflections.

The next step is to determine where the color clusters of interface reflections belong. In the $\mathbf{S}$ space, it is possible to determine that the color points in the region $\alpha_{S 1}$ belong to the object $\alpha$, since $\alpha_{S 1}$ has the decreased saturation values compared to $\alpha_{B}$ and does not overlap $\beta_{B}$ or $\beta_{S}$. Under neutralized illumination, interface reflections decrease the saturation values, and it is not probable that $\alpha_{S 1}$ belongs to the object $\beta$ because of the increased saturation values.

In determining where the regions $\alpha_{S 2}$ and $\beta_{S}$ belong, only spatial proximity is used as a clue. In the example shown in Fig. 19(e), $\beta_{S}$ is connected to $\beta_{B}$, and $\alpha_{S 2}$ has more boundaries with $\alpha_{S 1}$ than with $\beta_{B}$. If the region 
$\alpha_{S 2}$ has the same amount of boundaries both with $\beta_{B}$ and with $\alpha_{S 2}$, it is not clear where $\alpha_{S 2}$ belongs either in the image or in the $\mathbf{S}$ space, and thus the region corresponding to $\alpha_{S 2}$ cannot be determined.

\subsection{Inter-Reflection}

As explained earlier, the interface-interface and the interface-body inter-reflections are indistinguishable from the direct interface and the body reflections, respectively. By using the algorithm described above, therefore, the interface-interface reflection can be detected and separated together with the direct interface reflections while the interface-body reflection belongs to the body reflection.

The body-interface and the body-body interreflections can change the hue and saturation values of direct body reflections. When the inter-reflections are weak, their color clusters in the $\mathbf{S}$ space slightly deviate from the linear clusters from direct body reflections. Strong body-interface and body-body inter-reflections, on the other hand, can form completely different color clusters in the $\mathbf{S}$ space. Below we present a modified version of the hue-saturation segmentation algorithm for detecting the weak body-interface and body-body inter-reflections.

- The algorithm of seed growing and region merging is applied for hue and saturation segmentation with the uniformity parameters for hue and saturation higher than the system noise level. As a result, inter-reflections with small deviation from the linear body reflection clusters are included in the segmented regions of uniform hue and saturation within the uniformity parameters. Small diffuse interface reflections are also included in the regions.

- If there are regions excluded from the uniformhue segmentation, they are strong first-body (bodyinterface or body-body) reflections, and they can be identified as such. These regions are not processed further.

- The excluded regions from the uniform-saturation segmentation are identified using the method for detecting interface reflections as described earlier.

- In each segmented region of uniform saturation, another stage of saturation segmentation is applied with the uniformity parameter determined by the system noise. This will exclude the small diffuse interface reflections and inter-reflections within the region.
The saturation value of the body reflections without any inter-reflection or interface reflections can be found from the newly segmented region. Interreflections and interface reflections can be detected by applying a saturation threshold determined by the system noise.

- Based on the saturation values of body reflections, determine the values of interface reflections and inter-reflections.

The above algorithm detects only weak first-body inter-reflections. When there are strong first-body inter-reflections with changes in hue above the hueuniformity parameter, they are segmented as separate regions by the hue-segmentation. The hue-uniformity parameter determines the boundary between the weak and strong first-body inter-reflections. We use the twostage saturation-segmentation since inter-reflections are usually diffuse and spatially extended. If the uniformity parameter is close to the system noise level, the saturation segmentation may result in many isolated body reflection regions and the algorithm should rely more on the spatial processing than on the spectral processing. Besides, the body reflections and the interreflections may not be clearly distinguished with the image segmentation technique because of the diffuseness of inter-reflections. To maintain the spatial and spectral coherence, therefore, the uniformity parameter is set high in the first saturation segmentation in each hue-segmented region. In the next stage, the body reflection value is found in some regions within the uniformity parameter determined by noise, and a threshold is used for detecting diffuse inter-reflections. In an image of real objects, it is very uncommon that interreflections change the hue values without any change of saturation valucs. In most cases, therefore, only the saturation threshold can detect inter-reflections as well as diffuse interface reflections.

\section{Experimental Results}

To test our model for diffuse interface reflections, we performed an experiment with a plastic object with a rough surface. Figure 20(a) (see color figures, p. 268) shows the color image of a plastic hand. The image was taken under a collimated incandescent light source, and the illumination was neutralized using a white reference plate. Our CCD-camera output is linear, and mcasurcments were mostly in the linear range of an eight-bit image digitizer. We determined our system 


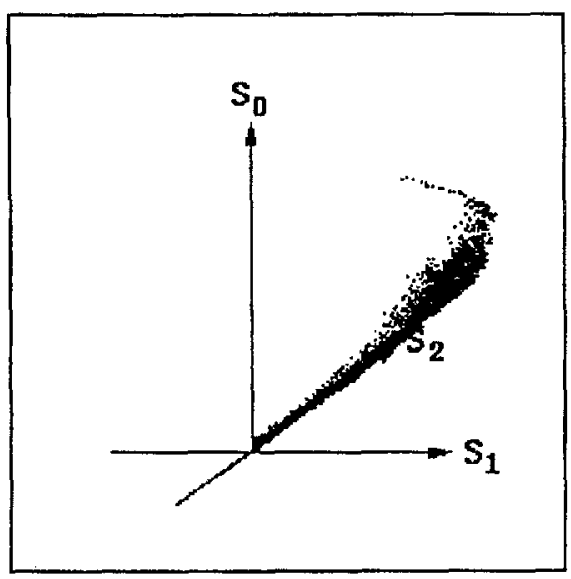

(a)

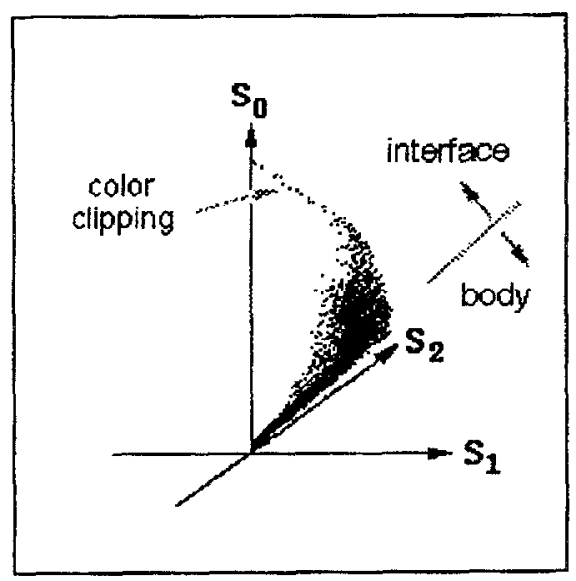

(b)

Figure 21. Color clusters from plastic hand in $\mathbf{S}$ space: (a) before illumination neutralization, (b) after illumination neutralization.

noise level experimentally with image data from many color-test plates, and the Fourier basis functions shown in Fig. 2(d) are used for the $\mathbf{S}$ space.

Figure 21(a) and (b) show the color clusters of the plastic hand before and after illumination neutralization, respectively. As expected, the color points from diffuse interface reflections form a planar cluster while shaded body reflections form a linear cluster. The deviations from the linear and planar clusters are due to the distortion and noise in the measurements, possible nonuniformity of the object color and inter-reflections. Note that the surface roughness of the object is not uniform and strong specular interface reflections are shown in the pixels that correspond to the smoother surfaces (the nails). Some specular interface reflections are clipped due to the limited dynamic range of the CCD sensor. Color clipping may occur in one, two or all three color bands (Shafer et al., 1990).

After the illumination neutralization, the interface reflections are aligned with the underlying body reflections in hue as shown in Fig. 21(b), and the saturation image after the illumination neutralization is shown in Fig. 20(b). The saturation of body reflections is high and uniform, and the reduced saturation indicates the presence of interface reflections. The saturation value of the body reflection was found by the saturation segmentation algorithm described in Section 4, and was used for detecting and separating interface reflections. Figure 20(c) and (d) show the separated body reflections, and the separated interface reflections, respectively. The image in Fig. 20 (d) is scaled up by 3 to show the diffuse interface reflections clearly. The results of separation are not correct in the pixels where color values are clipped.

To test our algorithm for multiple objects, we carried out an experiment with colored plastic balls. Four incandescent lights were used as illumination sources in two directions (right and left sides). They were made spatially extended and diffuse to produce extended and diffuse interface reflections and to reduce the contrast between interface and body reflections. Figures 22(a) and (b) (see color figures, p. 268) show the color image and saturation image of plastic color balls after the illumination neutralization, respectively. The color balls have very smooth surfaces and the green base has a rough surface. Figure 23 show the color clusters in the $S$ space after illumination neutralization. Although the surfaces of the plastic balls are smooth, the color points from interface reflections form planar clusters since the illumination is extended and diffuse. Because of the i1lumination neutralization, the interface reflections are aligned in hue with the underlying body reflections. This can be seen in the hue histogram in Fig. 24.

Hue segmentation by the algorithm of seed growing and region merging is shown in Fig. 25. Figure 25(a) and (b) show the hue values, and many seven-by-seven seed regions, respectively. The seeds are randomly but evenly located and they grow independently as far as the hue value of each region is within a uniformity parameter. The uniformity parameter is set higher than 


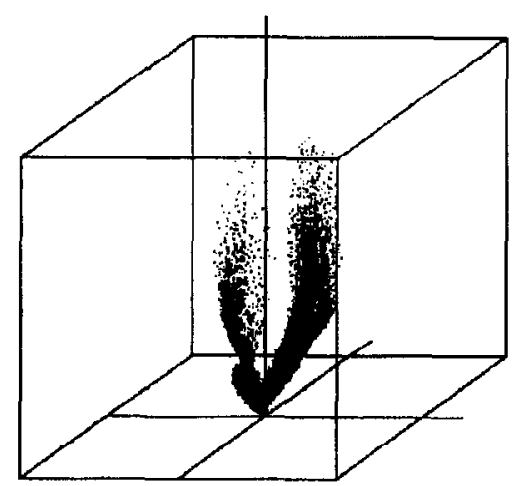

(a)

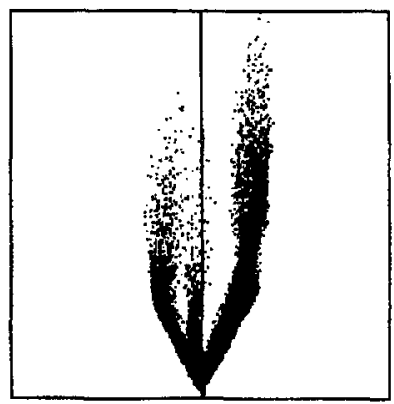

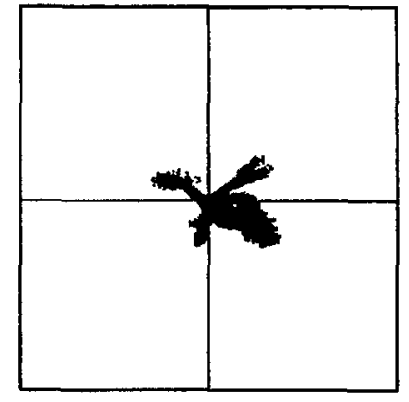

(b)

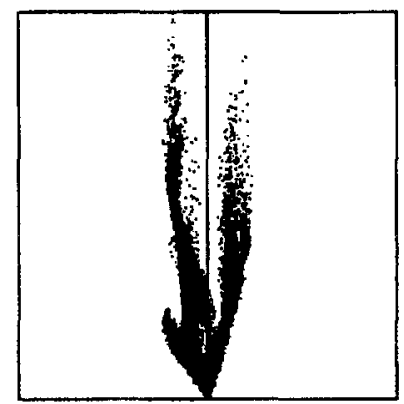

(d)

Figure 23. Color clusters from color balls: (a) $\mathbf{S}$ space, (b) top view $\left(S_{1}-S_{2}\right)$, (c) front view $\left(S_{1}-S_{2}\right)$, (d) side view $\left(S_{1}-S_{2}\right)$.

the noise level prior to the segmentation so that the huesegmented regions can include inter-reflections with small deviations in hue. The grown regions are merged when they are close in hue values, and the final segmentation of uniform-hue regions is shown in Fig. 25(c).

After the hue segmentation, saturation segmentation is performed with the same algorithm for each hue-segmented image. The uniformity parameter for the saturation segmentation has also been set higher than the noise level so that the saturation-segmented regions can include inter-reflections and diffuse interface reflections with small deviations in saturation. Figure 26(a) shows the saturation image of the red and the pink plastics balls that are segmented together due to their similar hue values. The seed regions are shown in Fig. 26(b) and the result of the seed-growing and region merging is shown in Fig. 26(c). The segmented regions in Fig. 26(c) have uniform saturation values within the uniformity parameter, and the excluded regions due to nonuniform saturation values are regarded 


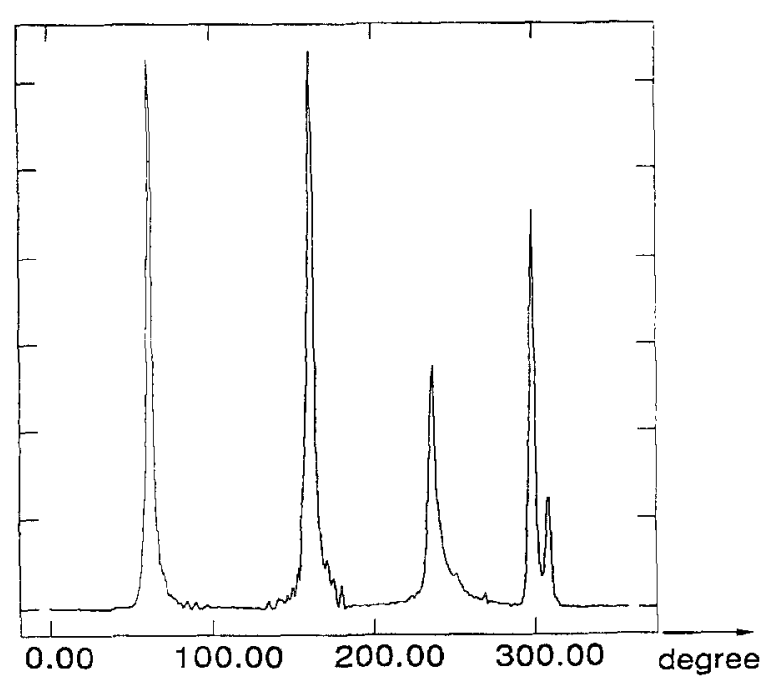

either as interface reflections or as inter-reflections. Figure 27(a) and (b) show the original hue-segmented image of the red and pink plastic balls and the segmented regions for uniform saturation, respectively, and Fig. 27(c) and (d) show the color clusters of the images (a) and (b) in the $S$ space, respectively. After the segmentation by saturation, the $S$ space is less crowded and the two planar clusters can be separated. The saturation histograms for the hue-segmented image of the red and pink plastic balls and for the segmented regions for uniform saturation are shown in Fig. 27(e) and (f), respectively.

For the regions excluded by the saturation segmentation, the information of spatial and spectral proximity determine to which object they belong as described in Section 4. Diffuse interface reflections and interreflections can be detected and separated by locally

Figure 24. Hue histogram.

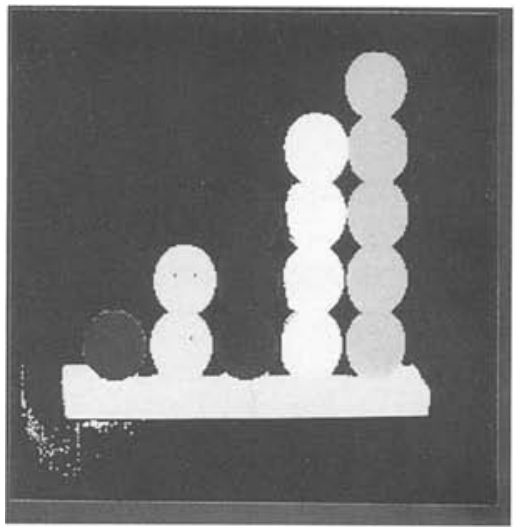

(a)

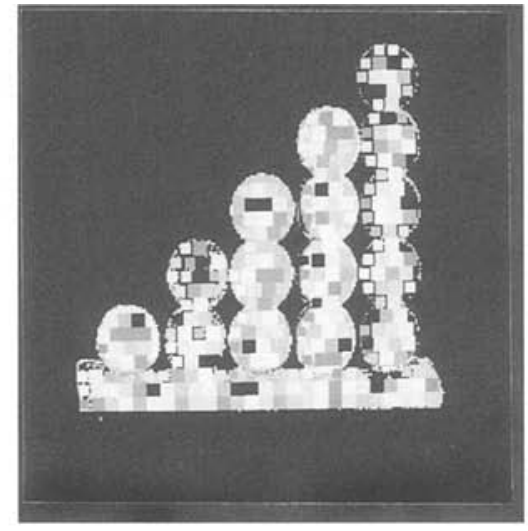

(b)

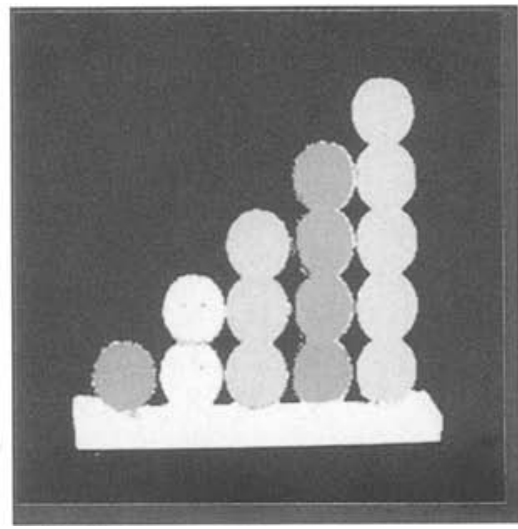

(c)

Figure 25. Segmentation by hue: (a) hue values, (b) seed regions, (c) segmented regions of uniform hue.

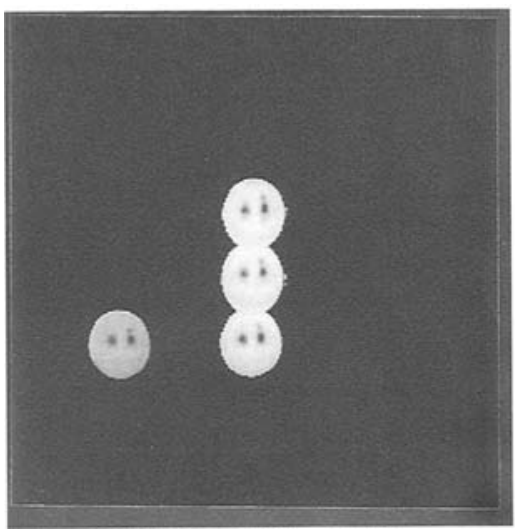

(a)

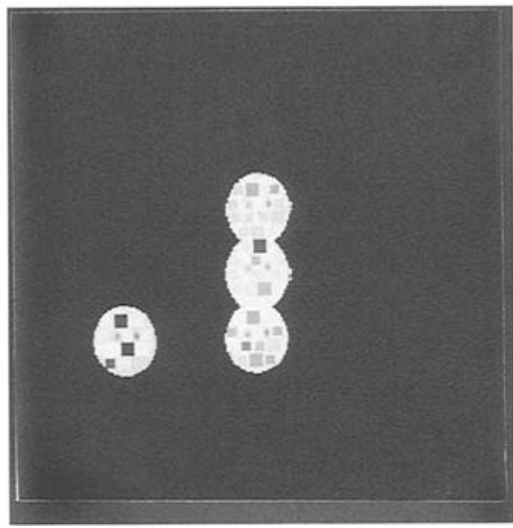

(b)

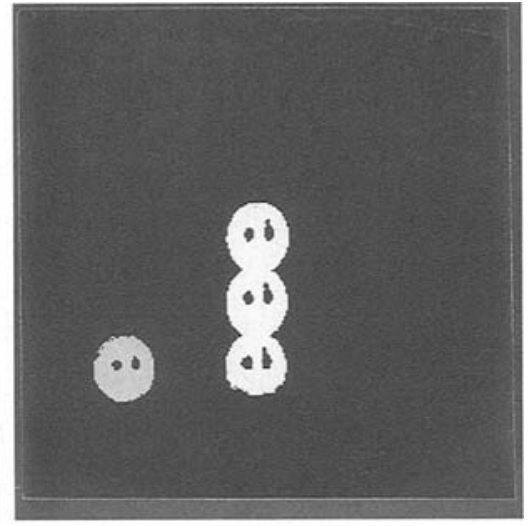

(c)

Figure 26. Segmentation by saturation: (a) saturation values, (b) seed regions, (c) segmented regions of uniform saturation. 


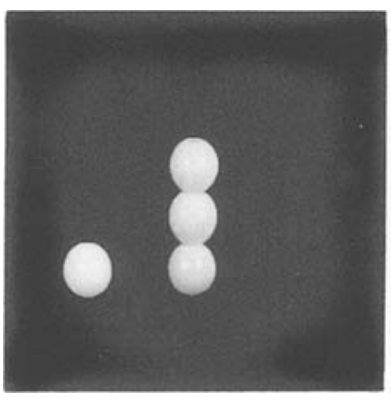

(a)

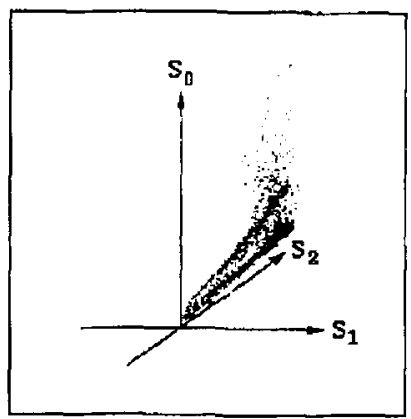

(c)

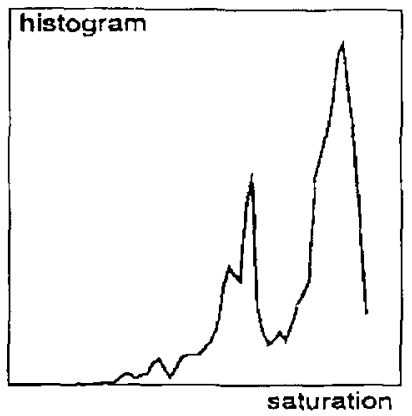

(e)

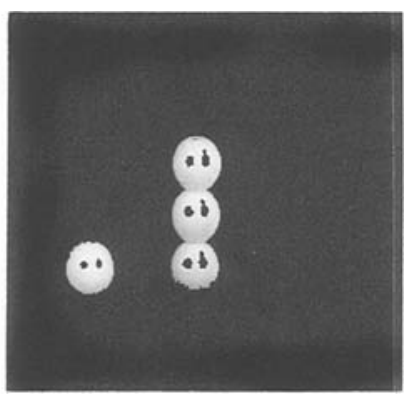

(b)

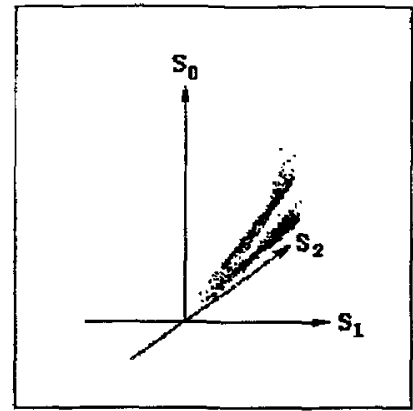

(d)

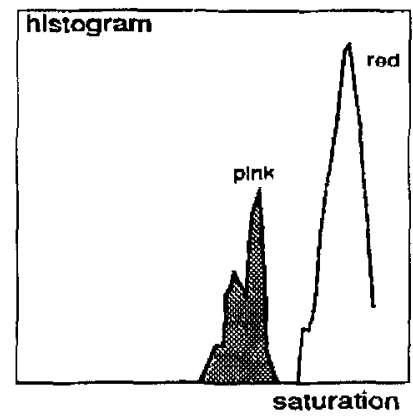

(f)

Figure 27. (a) Red and pink balls after hue segmentation, (b) scgmented regions of uniform saturation, (c) color clusters of red and pink balls in the $\mathbf{S}$ space, (d) color clusters of the saturation-segmented regions, (e) saturation histogram for red and pink balls, (f) saturation histogram for saturation-segmented regions.

thresholding saturation values within each region. The saturation value of the body reflections of uniform saturation is found by another stage of segmentation with a uniformity parameter determined by the system noise level. Figure 22(d) shows the separated interface reflections and inter-reflections from all the objects. This image is scaled up by 2 to show clearly the small diffuse interface reflections and inter-reflections. Note that the green base has very diffuse interface reflections and inter-reflections. Most of the inter-reflections between the color balls are due to the interface-interface reflections. The separated body reflections are combined and shown in Fig. 22(c).

Another experiment was carried out for a scene which consists of a red plate with a very smooth surface and yellow blocks with much rougher surfaces. Again, four diffused incandescent lights were used for 
illumination, and the global scene illumination was estimated with a reference plate. Figures 28(a) and (b) (see color figures, p. 268) show the color image and saturation images after illumination neutralization, respectively. In Fig. 28(b), it can be seen that the saturation values in the areas of interface reflections and inter-reflections are decreased while the saturation values in the shadowed area are kept high. Because of the smooth surface, the red plate shows mirror-like interreflections from the yellow blocks, and there are diffuse interface reflections on the yellow blocks.

Figure 28(c) shows the separated body reflections, and Fig. 28(d) shows the separated interface reflections and inter-reflections. The image in Fig. 28(d) is scaled up by 2 to show the small inter-reflections clearly. In Fig. 28(d), it can be seen that multiple reflections are strong in the wedge region where the red plate and the long block meet, and that the interface reflections on the yellow blocks are diffuse. These are not clearly visible in the original image before separation. When the body reflections removed, the objects in Fig. 28(d) look like metals showing only interface reflections and inter-reflections.

\section{Discussion}

The experimental results show that the scparation of diffuse interface reflections and inter-reflections is successful under the condition that a scene consists of surface patches of uniform hue and saturation and scene illumination is singly colored. We have experimentally determined the following parameters before applying the segmentation algorithm: two uniformity parameters for hue and saturation segmentation stages and a saturation threshold for detecting and separating diffuse interface reflections and inter-reflections. While the uniformity parameters are greater than the system noise level to include inter-inflections in a segmented region, the saturation threshold is decided by the noise level. In our system, the dominant source of noise is the electronics in the camera and digitizer, and measurement nonlinearity and quantization noise are found to be below the electronics noise level. We remove dark regions in a color image by a brightness threshold which is a little greater than the system noise level. If the brightness of a color pixel is low and comparable to the system noise level, there can be large errors in the calculation of hue and saturation values. Dark color pixels can result from a surface of low reflectance (black) and from a shadowed surface. We also remove the regions of low saturation since the color information is not reliable where saturation is low.

\subsection{S Space and Illumination Neutralization}

It is the combination of the illumination neutralization step and $\mathbf{S}$ space that mainly differentiates our model and algorithm from others. The $\mathbf{S}$ space is constructed to exploit maximally the NIR model and illumination neutralization. Although we used only the Fourier basis functions for the $\mathbf{S}$ space, we also suggested a method of using other basis functions that may represent natural colors better. The useful properties of the $\mathbf{S}$ space are that $S_{0}$ represents the neutral spectrum and total spectral power, and it is orthogonal to $S_{1}$ and $S_{2}$. With illumination neutralization, $S_{0}$ represents the spectral direction of scene illumination and interface reflection. Therefore, the presence of an interface reflection results in a change of color along the $S_{0}$ direction in the $S$ space, and only the saturation value of the underlying body reflection changes. On the other hand, hue values are independent of the presence of interface reflections since the hue is defined only with $S_{1}$ and $S_{2}$, which are orthogonal to $S_{0}$.

In analyzing surface reflections, we mainly used the $\mathbf{S}$ space since it is a linear space in terms of scalar scaling and vector addition and the color values that are the direct approximations of spectral curves. However the results of the analyses are explained in the $\hat{\mathbf{S}}$ space and in terms of hue and saturation which are used for our segmentation algorithm. The hue and saturation are the polar coordinate values in the $\hat{\mathbf{S}}$ space.

We found that the simple method of using a white reference plate for estimating scene illumination is very effective (Bajcsy et al., 1990). If a color chart with many known reflectances is used, however, the SPD of illumination can be even better approximated up to higher-order harmonics, and therefore the computation of surface reflection SPD can be more accurate. Novak and Shafer (1990) proposed a method for estimating scene illumination more precisely using a color chart of many known reflectances. When a reference object of known reflectance is not available or cannot be applied to a scene, spectrally neutral interface reflections from dielectrics can also be used as a reference for estimating scene illumination. Some algorithms have been proposed by H.C. Lee (1986) and Tominaga and Wandell $(1989 ; 1990)$ for estimating scene illumination using two or more interface reflections that are added to different body reflections. For those algorithms, at 


\section{COLOR FIGURES}

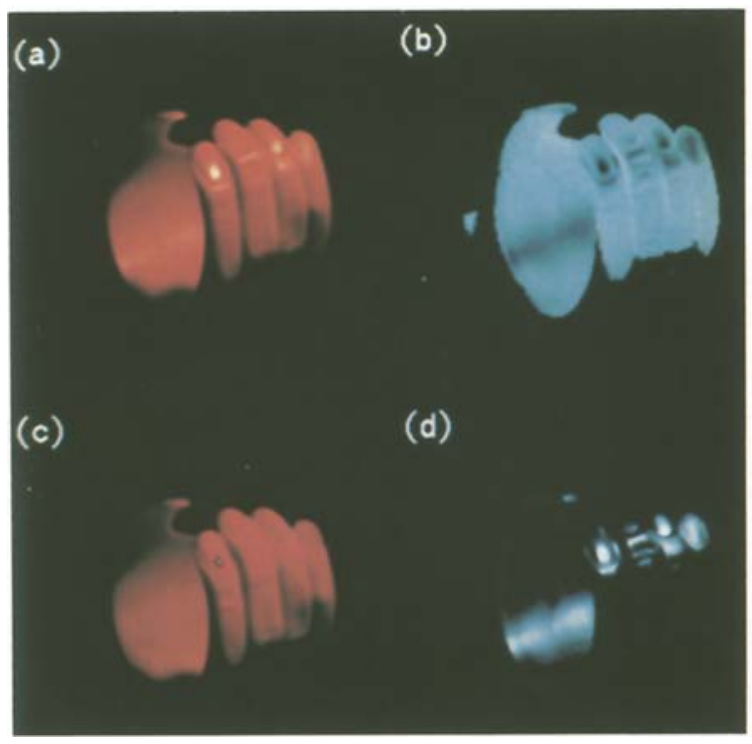

Figure 20. (a) Total reflection, (b) saturation, (c) body reflection (d) interface reflection.

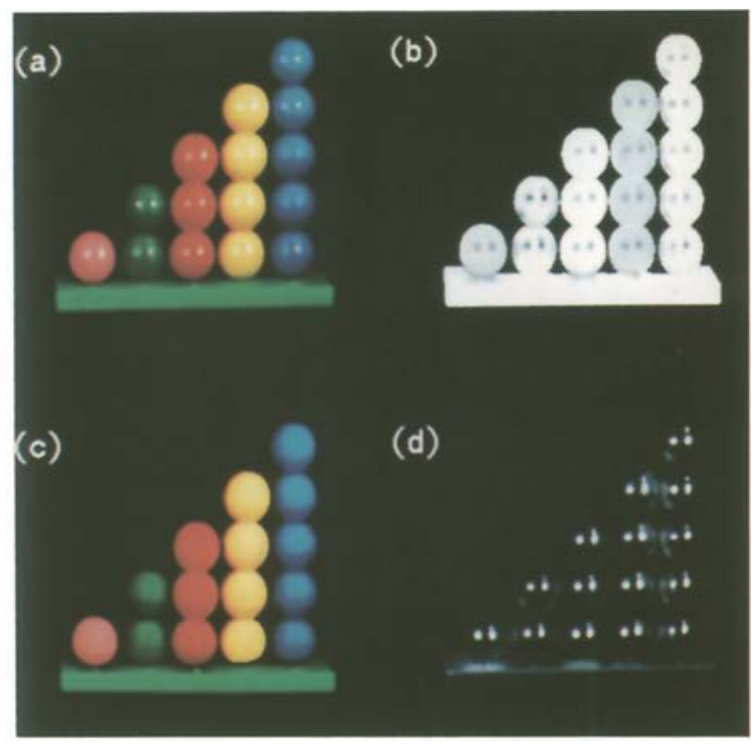

Figure 22. (a) Total reflection, (b) saturation, (c) body reflection (d) interface reflection and inter-reflection.

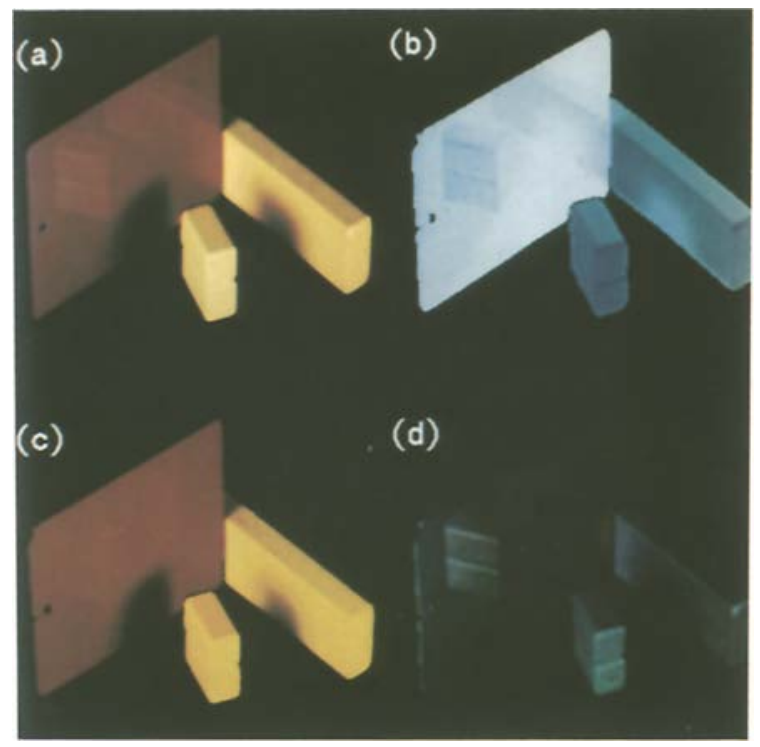

Figure 28. (a) Total reflection, (b) saturation, (c) body reflection, (d) interface reflection and inter-reflection. 
least two object regions with interface reflections are required since the interface reflections are not directly measurable but observed with underlying body reflections. If those algorithms work successfully, we do not need a reference object for our algorithm. However, we have not yet tested the algorithms for our images.

\subsection{Comparison with Other Research}

There have been several algorithms for the detection or separation of interface reflections using color and the dichromatic model. Shafer, Klinker, and Kanade (1990) developed an algorithm that segments and separates interface reflection in the three-dimensional RGB color space by finding body and interface reflection clusters based on their skewed $T$ model, and showed impressive results for dielectric materials. The skewed $T$ model is valid when interface reflections are specular. In this case, interface reflections form a linear cluster attached to a body reflection cluster as explained earlier. When interface reflections are diffuse, however, the interface reflections are distributed over some area of differently shaded body reflections, and form a skewed $P$ shape in general as shown in Section 3 (Bajcsy et al., 1990; Lee, 1991). Although the algorithm by Shafer et al. (1990) is able to detect small amounts of diffuse interface reflections which belong to the skewed $T$, the algorithm may not be effective for detecting diffuse interface reflections which generally do not form skewed $T$ clusters with body reflections. Recently Novak and Shafer (1992) proposed a method for analyzing color variation from rough surfaces using a color cluster model similar to the skewed $P$ model.

When interface reflection is diffuse due to a rough surface or extended illumination, the distribution of interface reflections in the skewed $P$ shape can be very sparse as shown in Sections 3 and 5. Therefore it is not easy to use the skewed $P$ shape in general as a model for clustering colors in a three-dimensional color space. Our algorithm finds linear body reflection clusters of uniform hue and saturation, and detects interface reflections which have the same color direction as that of estimated illumination. In principle this processing can be done in a three-dimensional space with the known illumination direction. However, our algorithm uses the decomposed one-dimensional values such as hue and saturation, since they exhibit a direct relationship with body and interface reflections when we align the interface reflections with the brightness axis in the $\mathbf{S}$ space by illumination neutralization.
There have also been approaches based on the use of some basis functions. Gershon (1987) suggested a color space, called C-space, based on a set of basis functions and color constancy (or illumination neutralization). The difference between the $\mathrm{C}$-space and the $\mathbf{S}$ space is that unlike the $\mathbf{S}$ space, the C-space is not oriented to the physically ncutral color direction (flat spectrum). Gershon's algorithm uses an image segmentation technique as a pre-processing stage and determines the reflection types of segmented regions by examining their color clusters in the $\mathrm{C}$-space based on the dichromatic model. The NIR property is used for verifying the interface reflection. The diffuse interface reflection is not incorporated into the model, and the algorithm assumes linear clusters both for interface and body reflections.

Healey and Binford have used a color space which is based on orthogonal basis functions and is normalized with respect to brightness (Healey, 1989; Healey and Binford, 1988a). However, their work is focused on object segmentation and material classification rather than on highlight detection from dielectric materials. They assume interface reflections are separated from dielectric materials before initial segmentation. In the normalized space, they segment a scene and classify materials.

Most of the previous work on inter-reflections assumes only body-body inter-reflections (Funt and Drew, 1991; Nayar et al., 1990b). Our method for detecting inter-reflections is based on our previous model of four reflection components: interface-interface, interface-body, body-interface and body-body reflections (Bajcsy et al., 1990). Novak and Shafer (1991) also analyzed inter-reflections based on the four components although a detection method was not suggested. Their analysis includes the body-interface and body-body reflections that show large deviations in color from direct body reflections.

\subsection{Limitations}

The major limitations of the algorithm come from three sources; two of them derive from assumptions and the other comes from image segmentation limitations:

- The assumption of uniform body reflectance in hue and saturation: This assumption is reasonable for many artificial and some natural objects. Many artificial objects are uniformly painted or dyed. Any regions with abrupt variation of hue and saturation 
would be segmented as distinct regions, and a scene comprised of such regions does not bring problems for image segmentation based on uniform hue and saturation values. However, any smooth and gradual variation in object reflectance above the noise level would be interpreted as interface reflections or as inter-reflection. Examples include color photographs, flowers, textiles, wood grains and any other granulated artificial or natural surfaces.

- The assumption of singly colored scene illumination: When there are strong primary illumination and some local inter-reflections, the assumption is valid. However, when there are several global illumination sources comparable in intensity but different in color, the variation in reflections due to the variation of illumination color can be detected as interface reflections or inter-reflections. An environment with strong inter-reflections from colored walls or ceilings, or an environment with more than one light source such as incandescent light, fluorescent light and daylight, does not satisfy the assumptions.

- Availability of good region information is not always guaranteed by image segmentation techniques. Any confusion in segmentation would result in a higher degree of confusion in the detection and separation of interface reflections.

\subsection{Direction of Research}

When the assumptions are satisfied, the algorithm approach presented in this paper detects and separates very diffuse interface reflections and inter-reflections that may not be easily visible in a color image before separation. For a small region in an image of a general scene, the assumptions of uniform reflectance and singly colored illumination are likely to hold, and our color model and algorithm presented in this paper can provide a useful way of interpreting local color variation and separating reflection components in a small image region. However finding such a region is not an easy task before detecting interface reflections.

We have previously proposed an algorithm which uses color and multiple views and detects specular in terface reflections and some diffuse interface reflections without relying on any of the above assumptions or on image segmentation techniques (Lee and Bajcsy, 1992). If specular interface reflection regions can be located prior to any image segmentation stages, a color image can be locally examined around the detected spccular interface reflections, and it may be possible to separate reflection components by locally applying the algorithm proposed in this paper. We are currently developing such an intergrated method.

\section{Conclusion}

In this paper, we proposed a reflection model and an algorithm for separating specular and diffuse interface reflections and inter-reflections from body reflections based on the dichromatic model, and experimental results are presented. Our model and algorithm further realize the potential of the dichromatic model. The major contributions are:

- the $\mathbf{S}$ space based on the three orthogonal basis functions where color clusters are analyzed simply using hue and saturation rather than three-dimensional color data,

- the illumination neutralization to align color clusters of interface reflections with the brightness axis in the S space.

The above allowed us to analyze the color clusters from diffuse and specular interface reflections. With illumination neutralization in the $\mathbf{S}$ space, it is observed that the presence of interface reflection does not change hue values of underlying Lambertian body reflections, but decreases only saturation values. Based on this observation and a region-based image segmentation technique, we developed an algorithm for the separation of interface reflections from body reflections. We also analyzed four different types of inter-reflections between objects: interface-interface, interface-body, bodyinterface and body-body reflections. We developed a technique to detect the interface-interface and weak body-interface and body-body reflections logether with interface reflections. The interface-body reflection is indistinguishable from the body reflection. Experimental results conform to our model and algorithm when objects in a scene consist of uniformly colored dielectrics under singly colored scene illumination.

\section{Acknowledgment}

This work was partly supported by E.I. du Pont de Nemours and Company, Inc. and partly by the following grants: Navy Grant N0014-88-K-0630, AFOSR Grants 88-0244, AFOSR 88-0296; Army/DAAL 0389-C-0031PRI; NSF Grants CISE/CDA 88-22719, 
IRI 89-06770, ASC 91 0813, and General Motors Research. Thanks to Alok Gupta and Jana Kosecka for providing an image segmentation algorithm and for helping us in incorporating it into our algorithm presented in this paper. Thanks also to Gareth Funka-Lea for his proof-reading. We are grateful to Paul Tannenbaum at DuPont for discussions and for providing us with the spectrophotometric measurements of color plates. We would like to thank Anthony Garito, Carl Grossman and Jung Won Wu in the Department of Physics for their help in the spectral measurements of camera and filters. We are also grateful to Richard Young at General Motors Research for discussions on many aspects of color science and applications. Special thanks to Steve Shafer at Carnegie Mellon University for helpful discussions and comments. We also thank the anonymous reviewers whose thoughtful comments greatly improved the clarity of this paper.

\section{References}

Bajcsy, R., Lee, S.W., and Leonardis, A. 1989. Computational aspects of color constancy. Technical Report, University of Pennsylvania, Philadelphia, PA.

Bajcsy, R., Lee, S.W., and Leonardis, A. 1990. Color image segmentation with detection of highlights and local illumination induced by inter-reflections. In Proc. 10th International Conf. on Pattern Recognition, Atlantic City, NJ.

Beck, J. 1967. Surface Color Perception. Cornell University Press: Ithaca, NY.

Beckman, P. and Spizzichino, A. 1963. Scattering of Electromagnetic Waves from Rough Surfaces. Pergamon Press: London, UK.

Blake, A. 1985. Specular stereo. In Proc. of 9th Int. Joint Conf. Artif. Intell., Los Angeles, CA, pp. 973-976.

Brelstaff, G. and Blake, A. 1988. Detecting specular reflections using lambertain constraints. In Proc. of IEEE Int. Conf. on Computer Vision, Tarpon Springs, FL, pp. 297-302.

Buchsbaum, G. 1980. A spatial processor model for object colour perception. J. Franklin Inst, 310:1-26.

Cohen, J. 1964. Dependency of the spectral reflectance curves of the munsell color chips. Psychon. Sci., 1:369-370.

Coleman, E.N. and Jain, R. 1982. Obtaining 3-dimensional shape of textured and specular surface using four-source photometry. Computer Graphics and Image Processing, 18:308-328.

D'Zmura, M. and Lennie, P. 1986. Mechanisms of color constancy. Journal of the Optical Society of America, 3:1662-1672.

Forsyth, D.A. 1988. A novel approach to colour constancy. Proceedings ICCV, 2:9-18.

Funka-Lea, G, and Bajcsy, R. 1993. Active color image analysis for recognizing shadows. In Proceedings of IJCAI-93, France.

Funt, B.V. and Drew, M.S. 1991. Color space analysis of mutual illumination. Technical report, Simon Fraser University, Burnaby, B.C., Canada.

Gershon, R. 1987. The Use of Color in Computational Vision. Ph.D. thesis, Department of Computer Science, University of Toronto.
Hanson, A.R. and Riseman, E.M. 1978. Segmentation for Natural Scenes. Academic Press: New York, NY.

Healey, G.H. 1989. Using color for geometry-insensitive segmentation. Journal of the Optical Society of America A, 6.

Healey, G.H. and Binford, T.O. 1988a. A color metric for computer vision. In Proceedings of CVPR, New York, NY, pp. 10-17.

Healey, G.H. and Binford, T.O. 1988b. Local shape from specularity. Computer Vision Graphics and Image Processing, 42.

Horn, B.K.P. 1986. Robot Vision. The MIT Press: Boston, MA.

Jepson, A.D., Gershon, R., and Tsotsos, J.K. 1986. Ambient illumination and the determination of material changes. Journul of the Optical Society of America A, 3(10):1700-1707.

Judd, D.B., MacAdam, D.L., and Wyszecki, G.W. 1964. Spectral distribution of typical daylight as a function of correlated color temperature. Journal of the Optical Society of America, 54.

Kanade. T. and Ikeuchi, K. 1991. Introduction to the special issue on physical modeling in computer vision. IEEE Trans. PAMI, 13:609-610.

Lee, H.-C. 1986. Method for computing the scene-illuminant chromaticity from specular highlights. Journal of the Optical Society of America, 3:1694-1699.

Lee, H.-C., Breneman, E.J., and Schulte, C.P. 1990. Modeling light reflection of computer vision. IEEE Trans. PAMI, 12:402-409.

Lee, S.W. 1991. Understanding of Surface Reflections in Computer Vision by Color and Multiple Views. Ph.D. thesis, University of Pennsylvania.

Lee, S.W. and Bajcsy, R. 1992. Detection of specularity using color and multiple views. Image and Vision Computing, 10:643-653.

Leonardis, A., Gupta, A., and Bajcsy, R. 1990. Segmentation as the search for the best description of the image in terms of primitives. In IEEE Proceedings of the Third International Conference on Computer Vision, Osaka, Japan, pp. 121-125.

Maloney, L.T. 1985. Computational approaches to color constancy. Technical report, Applied Psychology Laboratory, Stanford University, Stanford, CA.

Maloney, L.T. 1986. Evaluation of linear models of surface reflectance with small number of parameters. Journal of the Optical Society of America, 3:29-33.

Maloney, L.T. and Wandell, B.A. 1986. A computational model of color constancy. Journal of the Optical Society of America, 1:29-33.

Nassau, K. 1983. The Physics and Chemistry of Color. John Wiley and Sons: New York, NY.

Nayar, S.K., Fang, X.S., and Boult, T. 1993. Separation of reflection components using color and polarization. In Proceedings of the DARPA Image Understanding Workshop, Washington, DC, pp. 1049-1060.

Nayar, S.K., Ikeuchi, K., and Kanade, T. 1990a. Determining shape and reflectance of hybrid surfaces by photometric sampling. IEEE Trans. Roho. Autom., 6:418-431.

Nayar, S.K., Ikeuchi, K., and Kanade, T. 1990b. Shape from interreflections. In Proceedings of ICCV, Osaka, Japan, pp. 2-11.

Nayar, S.K., Ikeuchi, K., and Kanade, T. 1991. Surlace reflection: Physical and geometrical perspective. IEEE Trans. PAMI, 13:611-634.

Novak, C.L. and Shafer, S.A. 1990. Supervised color constancy using a color chart. Technical report, Carnegie Mellon University, Pittsburgh, PA. 
Novak, C.L. and Shafer, S.A. 1991. Anatomy of a histogram. Technical Report CMU-CS-91-203, Carnegie Mellon University, Pittsburgh, PA.

Novak, C.L. and Shafer, S.A. 1992. Anatomy of a color histogram. In Proceedings of CVPR, Champaign, II, pp. 599-605.

Ohlander, R., Price, K., and Reddy, D.R. 1978. Picture segmentation using a recursive region splitting method. Computer Graphics and Image Processing, 8:313-333.

1953. The Color Science. Optical Society of America. Thomas Y. Crowell Co.: New York, NY.

Oren, M.O. and Nayar, S.K. 1993. Generalization of the lambertian model. In Proceedings of the DARPA Image Understanding Workshop, Washington, DC, pp. 1037-1048.

Park, J.S. and Tou, J.T. 1990. Highlight separation and surface orientations for 3-d specular objects. In Proc. of IEEE Int. Conf. on Pattern Recog., Atlantic City, NJ, pp. 331-335.

Shafer, S.A. 1985. Using color to separate reflection components. COLOR Research and Application, 10:210-218.

Shafer, S.A., Klinker, G.J., and Kanade, T. 1990. A physical approach to color image understanding. Intern. Journal of Computer Vision, 4.

Tagare, H.D. and deFigueiredo, R.J. 1990. Simultaneous estimation of reflectance map and surface normal using photometric stereo. In Proc. of IEEE Int. Conf. on Computer Vision.
Tagare, H.D. and deFigueiredo, R.J. 1991. Photometric stereo for diffuse non-lambertian surface. IEEE Trans. PAMI, 13.

Tominaga, S. and Wandell, B. 1989. Standard surface-reflectance model and illuminant estimation. Journal of the Optical Society of America, 6.

Tominaga, S. and Wandell, B. 1990. Component estimation of surface spectral reflectance. Journal of the Optical Society of America, 7.

Torrance, K.E. and Sparrow, E.M. 1967. Theory for off-specular relfection from roughened surfaces. Journal of the Optical Society of America, 57:1105-1114.

Wandell, B.A. 1987. The synthesis and analysis of color images. IEEE Trans. on PAMI, 9:2-13.

Wolff, L.B. 1989. Using polarization to separate reflection components. In Proc. of IEEE Conference on Computer Vision and Pattern Recognition, San Diago, CA, pp. 363-369.

Wolff, L.B. 1991. Polarization Methods in Computer Vision. Ph.D. thesis, Department of Computer Science, Columbia University

Wolff, L.B. 1993. Diffuse and specular reflection from dielectric surfaces. In Proceedings of the DARPA Image Understanding Workshop, Washington, DC, pp. 1025-1030.

Wyszecki, G. and Stiles, W.S. 1967. Color Science. John Wiley and Sons: New York, NY. 